\title{
Static Prediction of Silent Stores
}

\author{
FERNANDO MAGNO QUINTÃO PEREIRA, UFMG, Brazil \& CNRS - LIRMM, France \\ GUILHERME VIEIRA LEOBAS, UFMG, Brazil \\ ABDOULAYE GAMATIÉ, CNRS - LIRMM, France
}

\begin{abstract}
A store operation is called "silent" if it writes in memory a value that is already there. The ability to detect silent stores is important, because they might indicate performance bugs, might enable code optimizations, and might reveal opportunities of automatic parallelization, for instance. Silent stores are traditionally detected via profiling tools. In this article, we depart from this methodology and instead explore the following question: is it possible to predict silentness by analyzing the syntax of programs? The process of building an answer to this question is interesting in itself, given the stochastic nature of silent stores, which depend on data and coding style. To build such an answer, we have developed a methodology to classify store operations in terms of syntactic features of programs. Based on such features, we develop different kinds of predictors, some of which go much beyond what any trivial approach could achieve. To illustrate how static prediction can be employed in practice, we use it to optimize programs running on nonvolatile memory systems.
\end{abstract}

CCS Concepts: $\bullet$ Software and its engineering $\rightarrow$ Compilers; $\bullet$ Hardware $\rightarrow$ Memory and dense storage; • Computing methodologies $\rightarrow$ Machine learning; $\bullet$ Information systems $\rightarrow$ Data mining;

Additional Key Words and Phrases: Silent stores, static analysis, machine learning, code optimization, nonvolatile memory

\section{ACM Reference format:}

Fernando Magno Quintão Pereira, Guilherme Vieira Leobas, and Abdoulaye Gamatié. 2018. Static Prediction of Silent Stores. ACM Trans. Archit. Code Optim. 15, 4, Article 44 (November 2018), 26 pages.

https://doi.org/10.1145/3280848

\section{INTRODUCTION}

Silent store is a term coined by Lepak and Lipasti (2000a) to denote a store operation that writes in memory a value that was already there. The ability to detect silent stores is important, because they might indicate performance bugs (Wen et al. 2017, 2018), might reveal opportunities for memorycentric code optimizations (Bouziane et al. 2018), and might enable automatic parallelization of software (Chabbi et al. 2018). Furthermore, at the hardware level, the detection of silent stores lets us bypass the usual limits imposed by data dependency hazards (Lepak and Lipasti 2000a;

This work has been made possible by the support of the following agencies: ANR (the CONTINUUM project: grant ANR15-CE25-0007-01) and CNPQ (grant PDE-202896/2017-0). Guilherme Leobas was supported by an MSc scholarship from CAPES.

Authors' addresses: F. M. Q. Pereira, UFMG, Brazil \& CNRS - LIRMM, France, Avenida Antônio Carlos, 6627, Belo Horizonte, MG, 31270-901; email: fernando@dcc.ufmg.br; G. V. Leobas, UFMG, Avenida Antônio Carlos, 6627, Belo Horizonte, MG, 31270-901, Brazil; email: guilhermel@dcc.ufmg.br; A. Gamatié, CNRS - LIRMM, 161 Rue Ada, Montpellier, 34090, France; email: abdoulaye.gamatie@lirmm.fr.

Permission to make digital or hard copies of all or part of this work for personal or classroom use is granted without fee provided that copies are not made or distributed for profit or commercial advantage and that copies bear this notice and the full citation on the first page. Copyrights for components of this work owned by others than the author(s) must be honored. Abstracting with credit is permitted. To copy otherwise, or republish, to post on servers or to redistribute to lists, requires prior specific permission and/or a fee. Request permissions from permissions@acm.org.

2018 Copyright is held by the owner/author(s). Publication rights licensed to ACM.

ACM 1544-3566/2018/11-ART44

https://doi.org/10.1145/3280848 
Subramaniam and Loh 2006), by the memory hierarchy (Dusser et al. 2009; Stone et al. 2005; Zhou et al. 2009), or by interthread dependencies (Rajwar and Goodman 2001).

Silent stores are traditionally detected via profiling tools, such as RedSpy (Wen et al. 2017), for instance. This approach is natural, given that "silentness," i.e., the occurrence of a silent store, is intuitively dependent on data. In this article, we depart from this methodology and instead, explore the following question: is it possible to predict silentness by analyzing the syntax of programs? The process of building an answer to this question is already interesting in itself, given the stochastic nature of silentness. Additionally, we believe that the static prediction of silent stores brings several benefits onto the already well-spread dynamic methodology: it is data independent; thus, it is more general and operationally simpler, as it does not require the pre-execution of programs.

In many ways, this work seeks to achieve, onto stores, the success that Ball and Larus (1993) and later $\mathrm{Wu}$ and Larus (1994) attained onto branches. Borrowing their words, our heuristics are "simple and require little program analysis." We also avow the same benefits they claimed over profiling: "Profiling-based prediction can be accurate, but it is inconvenient and time-consuming to use" (Ball and Larus 1993, p. 300). Yet, similarities stop there: stores and branches are very different operations. Reusing branch prediction heuristics would not be reasonable. Furthermore, 25 years of improvement on machine learning let us apply onto our problem techniques that were not common at the time. Thus, we use one order of magnitude more features to guide prediction and 10 times more benchmarks to validate it. Due to so many features, combining them via the same techniques employed in branch prediction would not do. Wu and Larus use the Dempster-Shafer (Dempster 1967; Shafer 1976) theory to combine heuristics. Combinations are evaluated in pairs; hence, just to use sets of two features, we would have thousands of new data points. Instead, we use statistical regression to predict silentness. This new methodology, coupled with the insights that we derive from it, let us bring forward a number of contributions:

- [Methodology]: Section 2 explains how we associate with each store operation a vector of features, and how we extract such features from a vast collection of benchmarks.

- [Insights]: Section 3 presents conclusions derived from our data. Some of these conclusions confirm folk knowledge, yet others are not obvious, even to experienced programmers.

- [Prediction]: We analyze different strategies to predict silentness in Section 4. These strategies range from trivial predictors to models based on nonlinear regression.

- [Application]: Section 5 explains how our predictor improves the usage of nonvolatile memory systems. Such systems, in which stores are more expensive than loads, have become trendy, due to recent concerns about energy optimization (Mittal and Vetter 2016).

As Section 2 explains, we have gathered a large collection of benchmarks to analyze silentness. Today, we can correctly predict different levels of silentness with a probability above $85 \%$. By levels of silentness we mean stores that are silent, for instance, $80 \%$ of the time. Nevertheless, this result must be understood with care: most of the stores in programs are not silent. Due to this heavy skewing, metrics such as precision and recall, which we revisit in Section 4, become extremely important. Under these metrics, we can discover about $63 \%$ of stores that are silent more than $80 \%$ of the time. Although our false-positive rate is nonnegligible, it is close to what can be expected from an optimal oracle and goes much beyond simple random guessing, as we discuss in Section 4 .

\section{METHODOLOGY}

We are interested in the prediction of silent stores, a problem stated in Definition 2.1. In Definition 2.1, we say that the syntactic occurrence of a store instruction in a program is a static instance of that operation. Its execution, i.e., when the program runs, is a dynamic instance. So, a static instance of a store can have several corresponding dynamic instances. 
Definition 2.1 (Static Prediction of Silent Stores). Input: a program $I$, a store instruction $\iota \in I$ (static instance), a real number $P \in[0,1]$. Output: classify $\iota$ as "silent" or "noisy." Validation: run $I$, and observe the behavior of each dynamic instance of $\iota$ :

- True Positive: $\iota$ is predicted silent, and its dynamic instances are silent more than $P$ times; that is to say, they are silent at least $(100 \times P) \%$ of times.

- True Negative: $\iota$ is predicted noisy, and its dynamic instances are silent less than $P$ times.

- False Positive: $\iota$ is predicted silent, but its dynamic instances are silent less than $P$ times.

- False Negative: $\iota$ is predicted noisy, but its dynamic instances are silent more than $P$ times.

Definition 2.1 asks for a static predictor; thus, we are not allowed to run the target program $I$, to base our prediction on the actual behavior of $\iota$. Instead, our decisions are guided by the syntactic features of the program $I$ that are related to the store $t$. Static program features are a notion that shall permeate this work; hence, we define them below:

Definition 2.2 (Static Program Feature). Given a program $I$ and a store instruction $\iota$, a static program feature $f(I, \iota)$ is any characteristic of $I$, related to $\iota$, with the following properties: [Binary]: $f(I, \iota) \in\{$ true, false $\}$; [Static]: $f(I, \iota)$ depends only on the syntax of $I$; [Consistent]: if $f(I, \iota)=x$, then $x$ is unique; [Available]: $f(I, \iota)$ can be computed in polynomial time.

The list of static features that we can extract from a program is virtually unbounded. Examples include the following: the store updates a pointer of floating-point type or the store happens within a doubly nested loop. In Section 2.2, we describe the features that we have used in this work. In this article, we use only binary features: either a store instruction presents the feature or it does not. In principle, we could let features range on countable sets. For instance, we could assume that Type is a feature with values in the set $\{$ int, char, float, double, etc $\}$. Instead, we have, for each type, a feature, such as is_int, is_char, is_float, is_double, etc. We adopted Boolean features because they simplify the prediction models that we later introduce in Section 4.

\subsection{Dataset}

Benchmarks. To validate the ideas discussed in this article, we have collected a number of benchmarks from well-known suites, such as SPEC CPU2006, MiBench, mediabench, BitBench, CoyoteBench, Trimaran, and so forth. In total, we reused 34 different collections, which gave us 222 programs to analyze. Most of our benchmarks have been taken from the LLVM's test suite (Lattner and Adve 2004); others were gathered from public repositories. Except for SPEC CPU2006, all these benchmarks are open-source software. In general, we have been able to reuse the benchmarks without having to change their source code. Some individual programs within particular benchmark suites had to be removed, due to our inability to compile or run them. Such shortcomings were rare, and are not related to any intervention related to our research on silent stores.

Profiler. We have implemented a profiler to evaluate the accuracy of predictions. This profiler, built on top of LLVM, lets us instrument store operations and count the number of occurrences of silent or noisy memory updates. Debugging information lets us map this information back into the program's source code. Our benchmarks, when run with their standard inputs under a timeout of 5 hours, gave us a total of 89,531 static instances of store operations that executed at least once. This number is slightly less than half the number of all the static instances of stores available in our benchmark collection. In total, we observed 689,376,922,141 dynamic instances of noisy stores, and 60,289,053,348 dynamic instances of silent stores; hence, the probability of a dynamic instance being silent is $8 \%$. However, if we average the probability of silentness of each individual store, then we obtain $19.23 \%$. This is the probability of a static instance being silent. 


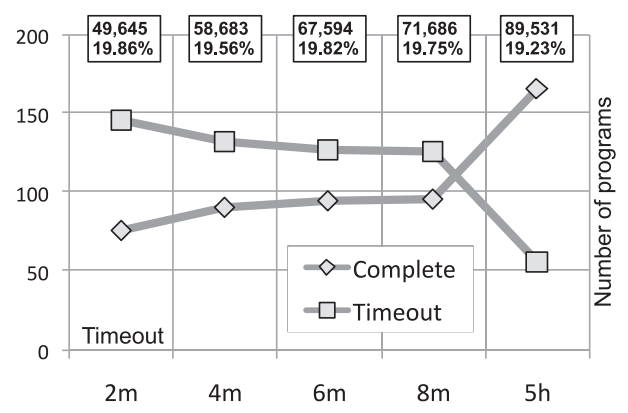

Fig. 1. Timeout versus successful termination.

Timeout. Profiling all the benchmarks that we have is a very time-consuming operation. Some of these benchmarks, such as Fhourstones, can take up to 1 hour to execute, depending on the input set we feed it. Thus, to render our framework practical, we adopted two strategies. First, we run the benchmarks in parallel, in a server with 12-core Intel(R) Xeon(R) E5-2620, at 2.00GHz. Second, we assign a timeout of 5 hours per benchmark. While parallelization bears no effect on our results, the timeout does, because it hinders us from observing every dynamic instance of the store instructions in our benchmarks. Figure 1 shows the number of benchmarks that finish successfully, depending on the timeout that we use. With a timeout of 2 minutes, we observe the termination of 76 programs, out of 222. With 8 minutes, 96 programs terminate. With 5 hours, this number goes up to 166 programs. The choice of 5 hours is arbitrary. Because we are not familiar with the behavior of every benchmark we have, we do not know if they terminate; hence, some sort of interruption would be necessary. We opted for a 5-hour timeout because it lets us finish the execution of one batch of tests in approximately 3 days, using 12 cores. As the reader shall find out, a considerable number of silent stores are related to initialization of data structures. Because data structures tend to be initialized at the beginning of the execution of programs, it is possible that the ratio of silent stores that we observe decreases with larger timeouts. However, we have not observed such behavior using shorter timeouts of 2, 4, 6, and 8 minutes. The boxes on top of Figure 1 show the number of static stores that executed in each experiment and the percentage of stores that were silent. The probability of silentness tends to remain within the range of $19.2 \%$ to $19.9 \%$.

Inputs. Some of our benchmarks contain multiple inputs. Nevertheless, we run them only once. We tried to choose the largest available input for each program: a task not always easy, given that some benchmarks lacked documentation. For SPEC CPU2006, we have used the "ref" input set, for instance. We have analyzed with Pin every one of our benchmarks, and all of them produce the execution of at least some binary instructions. In our collection, we found eight benchmarks that did not cause the execution of any store operation, such as Prolangs-C/plot2fig. We still distribute these benchmarks in our dataset, as they can be used to build histograms such as those that will be seen in Figures 10 and 17. This said, we have not used them in this article.

\subsection{The Feature Zoo}

We have collected 127 features associated with store instructions. To describe these features, we shall refer to the general format of a store operation as " $\ell: \star p=v$ ". This syntax denotes an instruction at the label $\ell$ in the program's text. This instruction indicates that a value in register $\mathrm{v}$ is uploaded into the location pointed by p. A collection of features is called a feature vector $F$. If $f$ is a feature, then $F(f, I, \iota)$ is the value of $f$ in $F$ with regard to instruction $\iota$ within program $I$. 
Intermediate Representation. We call feature mining the act of extracting, from the syntax of programs, features related to different store instructions. The software that performs such an action is a feature miner. We have implemented our feature miner in LLVM; therefore, we analyze the intermediate representation (IR) of programs. One important requirement of our feature miner is the ability to map stores in the LLVM IR back into source code, so that we can visually scrutinize interesting results. To meet this requirement, we made two decisions. First, features are mined when files are compiled, before link time. Second, we do not apply any optimization onto the programs that we analyze, except mem2reg.

Mem2reg is an LLVM module that converts programs to Static Single Assignment (SSA) form (Cytron et al. 1991). The SSA transformation approximates the syntax of C normally expected to denote memory references to the stores found in the LLVM intermediate representation. In this format, the following constructs of the $\mathrm{C}$ language yield values stored in memory: global variables (including function addresses); local variables whose addresses have been taken; constants allocated in memory such as strings; and memory aggregates (structs, unions, and arrays). On the other hand, local variables of scalar type are not stored in memory; rather, they can be understood as values allocated into registers. Notice that the use of mem2reg reduces the number of store operations in programs. On average, we have observed that if we convert a program to the SSA form, then we eliminate between three and four times the number of stores in its LLVM representation.

Example 2.3 (Intermediate Representation). Each example in Figure 4 gives our feature miner exactly one store, highlighted in gray. Local variables of scalar types are not stored in memory. Thus, variable p in Figure 4(b) and variable i in Figure 4(c) are not written via store operations.

2.2.1 Categories of Features. We have organized our 127 features into eight categories. Out of the 127 features, 100 have been observed in our benchmarks. Figure 3 shows them, together with the probability of silentness associated with their occurrence. Features in each category refer to different parts of the store instruction " $\ell: \mathrm{p}[\mathrm{i}]=\mathrm{v}$ " and are described as follows:

- ValueType. The type of variable v: Vfp: 32-bit floating point; Vdb: 64-bit floating point; Vin: integers (with different bitwidths); Vpt: pointer.

- VAlueSize. The size of the type, in bits: sz0: size is unknown v; sz1: 1 bit; sz8: 2 to 8 bits; s16: 9 to 16 bits; s32: 16 to 32 bits; szN: more than 32 bits.

- VAlueDeps. The operands (instructions, addresses, and constants) that contribute to form the value of v. Figure 2 shows the 32 features in this category.

- ValueOrigin. The last instruction or operation that produced the value v. Figure 2 also shows the 18 features observed in this category.

- PointerType. The type of p: Pfp: 32-bit floating point; Pdb: 64-bit floating point; Pin: integer; Pst: C-like struct; Pay: array; Ppt: another pointer; Pvc: SIMD vector.

- PointerLoc. The location of the region pointed by p: Msc: static memory; Msk: stack; Mhp: heap; Mar: unknown location loaded from function argument; Mfn: unknown location returned by function; Mph: unknown location loaded from an SSA $\phi$-function; Mbt: unknown location produced by a bitwise operation; Mld: other unknown locations.

- LABELLoc. The location $\ell$ of the instruction within the program. Smn: within the main function; S10: not inside a loop. S11-S13: within a singly, doubly, or triply nested loop; S1x: within more than three loop nests. Scm: $\ell$ postdominates the entry point of the function. Thus, if this function is invoked, the store happens compulsorily. Scl: $\ell$ is compulsory within the loop where it is located. Spl: $\ell$ exists within a compulsory loop, i.e., the loop's entry point postdominates the function's entry point. $S 1 x: \ell$ is within a single-exit loop; i.e., 


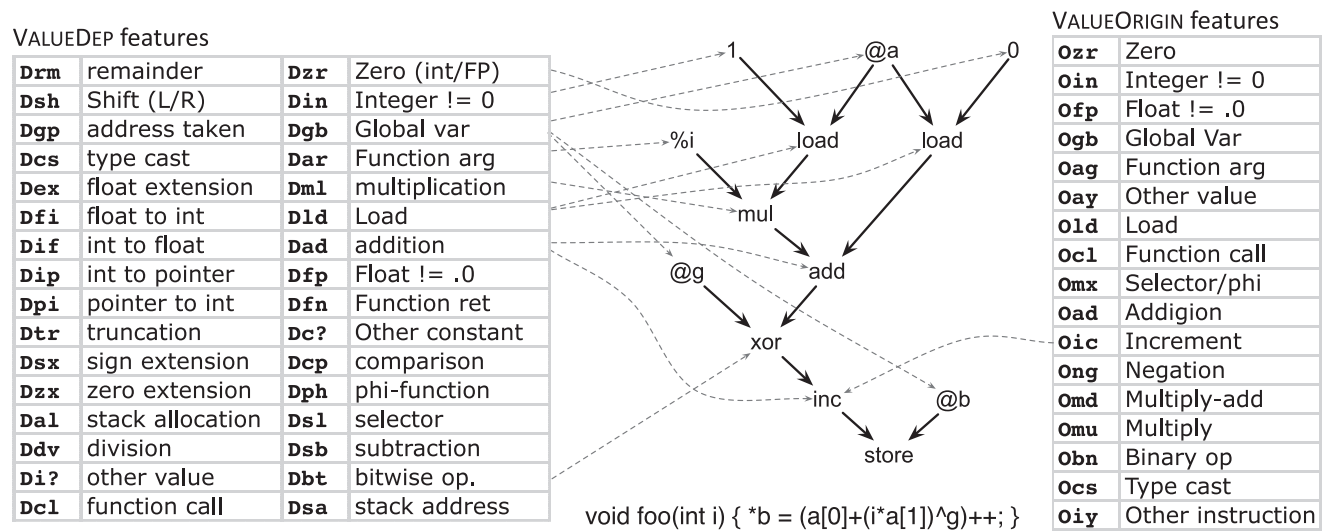

Fig. 2. List of VALUEDEPS and VALUEOrigin features, with a code example. The directed acyclic graph refers to function foo, whose source code is given on the bottom of the figure. Variables a, b, and g are global.

after the loop runs, the program always reaches the same point. Ssl: $\ell$ is within a singlelatch loop. A latch is a block inside the loop that leaves it. Sdl: $\ell$ dominates the single latch in the loop. Smp: in a basic block with multiple predecessors. Sms: in a basic block with multiple successors. Svi: reached only by invariant definitions of v. Spi: reached only by invariant definitions of $\mathrm{p}$.

- PointerStride. The offset $\mathrm{i}$ that is added to $\mathrm{p}$ when building the store address, e.g., $\mathrm{p}+$ $i$. We use LLVM's scalar evolution to recover this information. Erc: $\mathrm{i}$ is created by some recursive expression, e.g., $\mathrm{i}=\mathrm{i}+1$; Eaf: $\mathrm{i}$ is created by some affine expression, e.g., $\mathrm{i}=$ $2 * b+c$; Es1: $i$ has a stride of size 1; Es4: $i$ has a stride of size 4; Es8: stride of size 8; EsN: regular stride, but its value is unknown; Esp: stride has the same size as the region pointed by p; Etp: i exists in a loop of known trip count.

Example 2.4 (Program Features). Figures 2 and 4 show features extracted for different programs. Notice the difference between features in the categories ValueDep and ValueOrigin. The former contains any instructions or operands that contributed to form a value. Thus, the same store instruction might be associated with several of them. For instance, the store in Figure 2 contains eight different features in the VALUEDEP group. Such features describe operations that are present in the program slice forming that instruction. On the other hand, features in the VALUEORIGIN category are always unique: a value can be produced by only one operation, which is the last variable or instruction in the program slice of that value.

2.2.2 Collecting Features. As mentioned in Definition 2.2, static program features must be Available. Availability means that all the elements active in a feature vector must be collectable in polynomial time, preferably by static analyses that are simple to implement and deploy. All the features that we use in this article have been collected using equipment already in place in the LLVM infrastructure. Figure 5 shows the runtime of our feature miner. For comparison, we show the runtime of LLVM passes used to compute the ranges of integer variables (implemented after Quintao Pereira et al. (2013)) and to build the program's dominance tree. These two compilation passes contribute to the overall runtime of our feature miner. To build Figure 5, we have randomly chosen 50 benchmarks among the open-source programs that we have and have sorted them by number of instructions. As Figure 5 shows, the process of collecting features is practical: we take 


\begin{tabular}{|c|c|c|c|c|c|c|}
\hline Total & Feat & P1.0 & P0.8 & P0.6 & P0.4 & P0.2 \\
\hline 1078 & $V f p$ & 0.13 & 0.24 & 0.28 & 0.32 & 0.38 \\
\hline 3116 & $\mathrm{Vdb}$ & 0.08 & 0.14 & 0.15 & 0.19 & 0.22 \\
\hline 27266 & Vin & 0.12 & 0.17 & 0.2 & 0.24 & 0.29 \\
\hline 7914 & Vpt & 0.1 & 0.12 & 0.14 & 0.17 & 0.19 \\
\hline 7915 & $\mathrm{sz0}$ & 0.1 & 0.12 & 0.14 & 0.17 & 0.19 \\
\hline 142 & sz1 & 0.33 & 0.47 & 0.54 & 0.61 & 0.65 \\
\hline 1118 & sz 8 & 0.16 & 0.23 & 0.27 & 0.32 & 0.37 \\
\hline 858 & s16 & 0.1 & 0.21 & 0.23 & 0.27 & 0.34 \\
\hline 24195 & s32 & 0.12 & 0.17 & 0.2 & 0.24 & 0.29 \\
\hline 5146 & $\mathrm{szN}$ & 0.09 & 0.15 & 0.17 & 0.2 & 0.23 \\
\hline 614 & $P f p$ & 0.1 & 0.23 & 0.26 & 0.3 & 0.37 \\
\hline 2087 & $\mathrm{Pdb}$ & 0.07 & 0.14 & 0.16 & 0.19 & 0.22 \\
\hline 18523 & Pin & 0.09 & 0.14 & 0.17 & 0.21 & 0.25 \\
\hline 10797 & Pst & 0.15 & 0.2 & 0.23 & 0.28 & 0.32 \\
\hline 3532 & Pay & 0.15 & 0.22 & 0.24 & 0.28 & 0.33 \\
\hline 3820 & Ppt & 0.08 & 0.1 & 0.1 & 0.12 & 0.14 \\
\hline 1 & Pvc & 0 & 1 & 1 & 1 & 1 \\
\hline 6281 & Ozr & 0.4 & 0.48 & 0.52 & 0.58 & 0.62 \\
\hline 3857 & Oin & 0.05 & 0.1 & 0.12 & 0.15 & 0.18 \\
\hline 466 & ofp & 0.02 & 0.03 & 0.03 & 0.07 & 0.08 \\
\hline 454 & Ogb & 0.09 & 0.15 & 0.18 & 0.22 & 0.27 \\
\hline 427 & Oay & 0.16 & 0.2 & 0.21 & 0.22 & 0.24 \\
\hline 5043 & Oag & 0.05 & 0.09 & 0.11 & 0.15 & 0.19 \\
\hline 5322 & old & 0.09 & 0.14 & 0.18 & 0.23 & 0.29 \\
\hline 2578 & $\mathrm{Ocl}$ & 0.11 & 0.15 & 0.18 & 0.24 & 0.28 \\
\hline 937 & Omx & 0.07 & 0.15 & 0.21 & 0.26 & 0.32 \\
\hline 4075 & Oad & 0.05 & 0.08 & 0.11 & 0.13 & 0.16 \\
\hline 3082 & Oic & 0 & 0.01 & 0.01 & 0.01 & 0.02 \\
\hline 140 & Ong & 0.04 & 0.16 & 0.2 & 0.22 & 0.23 \\
\hline 9 & Ont & 0 & 0 & 0.11 & 0.11 & 0.22 \\
\hline 768 & Omd & 0.07 & 0.17 & 0.18 & 0.22 & 0.25 \\
\hline 938 & Omu & 0.04 & 0.11 & 0.14 & 0.17 & 0.22 \\
\hline 989 & Obn & 0.04 & 0.12 & 0.16 & 0.2 & 0.23 \\
\hline 2728 & Ocs & 0.05 & 0.09 & 0.12 & 0.17 & 0.22 \\
\hline 12 & Oal & 0 & 0 & 0 & 0.08 & 0.08 \\
\hline 238 & Oun & 0.04 & 0.1 & 0.13 & 0.15 & 0.17 \\
\hline 1030 & Oiy & 0.01 & 0.03 & 0.03 & 0.05 & 0.06 \\
\hline 5306 & Msc & 0.19 & 0.23 & 0.25 & 0.26 & 0.28 \\
\hline 18978 & Msk & 0.08 & 0.12 & 0.15 & 0.19 & 0.22 \\
\hline 761 & Mhp & 0.16 & 0.22 & 0.24 & 0.28 & 0.3 \\
\hline 6127 & Mar & 0.11 & 0.16 & 0.19 & 0.24 & 0.28 \\
\hline 6075 & Mld & 0.14 & 0.22 & 0.26 & 0.31 & 0.36 \\
\hline 1190 & Mfn & 0.15 & 0.2 & 0.23 & 0.25 & 0.28 \\
\hline 930 & $\mathrm{Mph}$ & 0.06 & 0.12 & 0.15 & 0.19 & 0.25 \\
\hline 7 & Mbt & 0 & 0 & 0.43 & 0.43 & 0.43 \\
\hline 18800 & Dzr & 0.18 & 0.24 & 0.28 & 0.32 & 0.37 \\
\hline 24733 & Din & 0.05 & 0.1 & 0.13 & 0.16 & 0.2 \\
\hline 1353 & $D f p$ & 0.03 & 0.08 & 0.1 & 0.15 & 0.17 \\
\hline 5181 & Dgb & 0.08 & 0.14 & 0.16 & 0.19 & 0.23 \\
\hline 5008 & Dfn & 0.09 & 0.14 & 0.17 & 0.21 & 0.25 \\
\hline
\end{tabular}

\begin{tabular}{|c|c|c|c|c|c|c|}
\hline Total & Feat & $\mathbf{1}$ & 0.8 & 0.6 & 0.4 & 0.2 \\
\hline 2844 & Dc? & 0.07 & 0.1 & 0.11 & 0.13 & 0.15 \\
\hline 11755 & Dar & 0.07 & 0.12 & 0.14 & 0.17 & 0.21 \\
\hline 20216 & Dld & 0.06 & 0.11 & 0.13 & 0.17 & 0.21 \\
\hline 5385 & $\mathrm{Dcl}$ & 0.09 & 0.13 & 0.16 & 0.2 & 0.24 \\
\hline 590 & Dcp & 0.11 & 0.19 & 0.24 & 0.35 & 0.39 \\
\hline 5185 & Dph & 0.07 & 0.15 & 0.19 & 0.23 & 0.28 \\
\hline 392 & Dsl & 0.05 & 0.09 & 0.13 & 0.19 & 0.24 \\
\hline 10883 & Dad & 0.04 & 0.09 & 0.11 & 0.14 & 0.17 \\
\hline 3336 & Dsb & 0.05 & 0.11 & 0.13 & 0.17 & 0.22 \\
\hline 3397 & Dml & 0.05 & 0.11 & 0.13 & 0.16 & 0.2 \\
\hline 1249 & Ddv & 0.02 & 0.09 & 0.13 & 0.17 & 0.22 \\
\hline 341 & Drm & 0.02 & 0.05 & 0.08 & 0.12 & 0.21 \\
\hline 1660 & Dsh & 0.02 & 0.09 & 0.12 & 0.15 & 0.2 \\
\hline 1397 & Dbt & 0.05 & 0.12 & 0.16 & 0.19 & 0.23 \\
\hline 13275 & Dgp & 0.06 & 0.12 & 0.15 & 0.18 & 0.23 \\
\hline 2233 & DCs & 0.04 & 0.08 & 0.12 & 0.14 & 0.16 \\
\hline 181 & Dex & 0.06 & 0.09 & 0.14 & 0.18 & 0.22 \\
\hline 207 & Dfi & 0.03 & 0.08 & 0.11 & 0.14 & 0.14 \\
\hline 648 & Dif & 0.03 & 0.07 & 0.11 & 0.15 & 0.19 \\
\hline 15 & Dip & 0.13 & 0.13 & 0.33 & 0.33 & 0.33 \\
\hline 86 & Dpi & 0.07 & 0.12 & 0.15 & 0.16 & 0.2 \\
\hline 1955 & Dtr & 0.04 & 0.09 & 0.12 & 0.18 & 0.22 \\
\hline 6876 & Dsx & 0.05 & 0.12 & 0.15 & 0.2 & 0.25 \\
\hline 1753 & Dzx & 0.09 & 0.16 & 0.19 & 0.24 & 0.29 \\
\hline 12290 & Dal & 0.05 & 0.09 & 0.12 & 0.16 & 0.21 \\
\hline 289 & Di? & 0.03 & 0.1 & 0.13 & 0.15 & 0.17 \\
\hline 1166 & Smn & 0.21 & 0.22 & 0.23 & 0.23 & 0.24 \\
\hline 25142 & S10 & 0.13 & 0.17 & 0.19 & 0.23 & 0.26 \\
\hline 8957 & Sl1 & 0.08 & 0.14 & 0.18 & 0.22 & 0.27 \\
\hline 3543 & $\mathrm{~S} 12$ & 0.08 & 0.17 & 0.21 & 0.24 & 0.29 \\
\hline 1274 & $\mathrm{~S} 13$ & 0.07 & 0.17 & 0.22 & 0.26 & 0.29 \\
\hline 458 & Sln & 0.06 & 0.11 & 0.15 & 0.21 & 0.27 \\
\hline 16197 & $\mathrm{Scm}$ & 0.13 & 0.17 & 0.19 & 0.23 & 0.26 \\
\hline 5830 & $\mathrm{Scl}$ & 0.1 & 0.15 & 0.19 & 0.24 & 0.29 \\
\hline 643 & Spl & 0.1 & 0.16 & 0.19 & 0.3 & 0.33 \\
\hline 11032 & Slx & 0.08 & 0.15 & 0.19 & 0.24 & 0.29 \\
\hline 6881 & Smp & 0.14 & 0.2 & 0.23 & 0.28 & 0.32 \\
\hline 10631 & Sms & 0.1 & 0.15 & 0.18 & 0.23 & 0.27 \\
\hline 13771 & Ssl & 0.08 & 0.15 & 0.19 & 0.23 & 0.28 \\
\hline 8741 & Sdl & 0.07 & 0.13 & 0.16 & 0.21 & 0.25 \\
\hline 3151 & Svi & 0.19 & 0.29 & 0.34 & 0.4 & 0.46 \\
\hline 6174 & Spi & 0.05 & 0.1 & 0.14 & 0.18 & 0.22 \\
\hline 2364 & Erc & 0.09 & 0.14 & 0.19 & 0.24 & 0.29 \\
\hline 2364 & Eaf & 0.09 & 0.14 & 0.19 & 0.24 & 0.29 \\
\hline 100 & Es 1 & 0.09 & 0.12 & 0.16 & 0.26 & 0.41 \\
\hline 565 & Es 4 & 0.05 & 0.14 & 0.21 & 0.29 & 0.35 \\
\hline 631 & Es 8 & 0.21 & 0.24 & 0.28 & 0.32 & 0.35 \\
\hline 952 & ESN & 0.04 & 0.1 & 0.13 & 0.17 & 0.2 \\
\hline 1122 & Esp & 0.05 & 0.12 & 0.18 & 0.25 & 0.31 \\
\hline 3181 & Etp & 0.08 & 0.19 & 0.24 & 0.29 & 0.33 \\
\hline
\end{tabular}

Fig. 3. Probabilities of silentness distributed per features. Total shows number of instructions that present each feature. The other columns show thresholds of silentness. Darker cells indicate higher proportion of silent stores. For instance, $48 \%$ of stores with the feature $0 z r$ were silent $80 \%$ or more of the time.

struct S $\{$ double $\mathrm{f} ;$;
struct S foo(double d) \{
struct S s0;
s0.f = d;
return s0;
\}
\begin{tabular}{|l|l|l|}
\hline Vdb & szN & rg 1 \\
\hline Pst & Oag & Msk \\
\hline Dar & S 10 & Scm \\
\hline
\end{tabular}

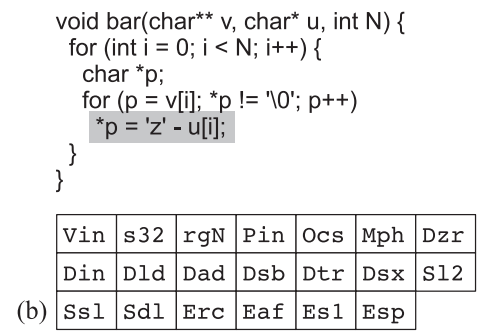

(b) \begin{tabular}{l|l|l|l|l|l|} 
Ssl & Sdl & Erc & Eaf & Es 1 & Esp \\
\hline
\end{tabular}

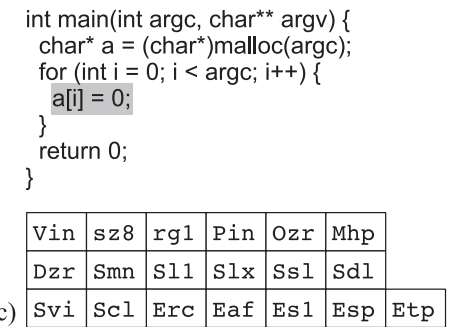

Fig. 4. Examples of static feature vectors extracted for three different programs. 


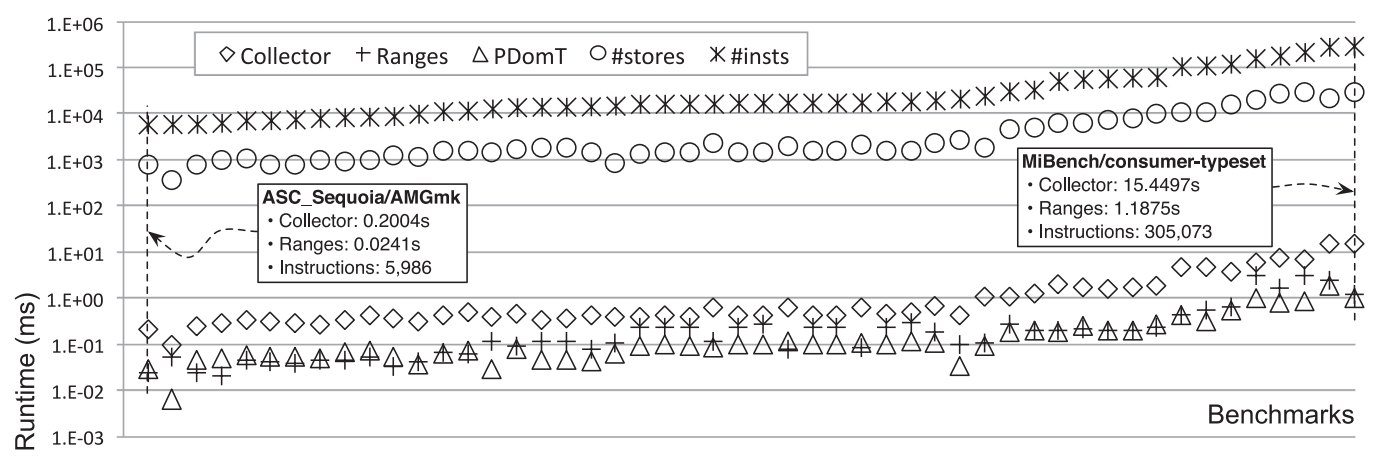

Fig. 5. Runtime of the feature miner used in this article. Each tick along the x-axis represents a different benchmark. Benchmarks are sorted by number of instructions in the LLVM intermediate representation. The $y$-axis shows time, in seconds. For the sake of comparison, we report the runtime of three different LLVM passes: Collector is the feature miner; Ranges is LLVM's range analysis (Quintao Pereira et al. 2013); PDomT constructs the program's dominance tree.

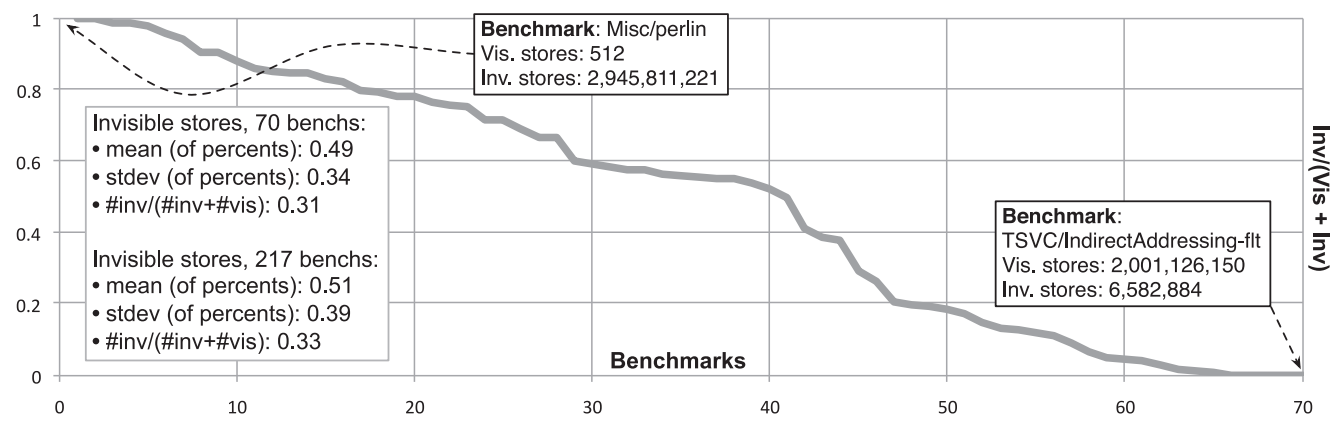

Fig. 6. Proportion of number of invisible stores (\#Inv) over the total number of stores observed via Pin (\#Vis + $\#$ Inv). The ratio \#Inv/(\#Vis + \#Inv) refers to the absolute number of dynamic instances of stores.

about 15 seconds to analyze consumer-typeset, a program with $305 \mathrm{~K}$ instructions on a $2 \mathrm{GHz}$ machine.

\subsection{On the Number of Analyzable Stores}

The data used in this work concerns visible instructions. A visible instruction is any instruction that exists in the source code of a program (Leobas et al. 2018). In contrast, invisible instructions are those either (1) inserted by the compiler, in the process of code generation, or (2) that belong to libraries dynamically linked against the final executable program. In the particular case of stores, invisible instructions abound in the first category. Stores are created by the compiler during spilling-the act of mapping variables into memory as a consequence of high register pressure.

To provide the reader with some idea on the ratio of visible and invisible instructions, we have used Pin (Luk et al. 2005) to count them. To separate visible from invisible instructions, we insert a dummy function around stores in the source code, via an LLVM pass. Figure 6 shows the proportion of invisible instructions in the 70 benchmarks in our collection with the largest number of dynamic store instances. We found that $34 \%$ of the dynamic instances of stores in these benchmarks are invisible. This proportion varies widely across benchmarks. If we average the percentage of invisible stores per benchmark, we obtain $51 \%$, with a standard deviation of $39 \%$. Some benchmarks 

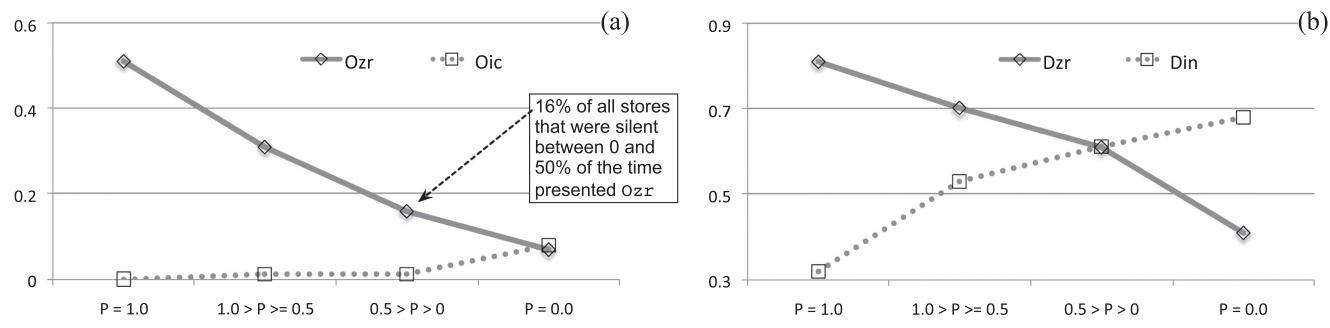

Fig. 7. Distribution of four features among different thresholds of silentness.

have barely any invisible instruction, whereas others only show this kind of operation. Figure 6 highlights two extreme cases. This experiment indicates two facts. First, the analyses that shall be discussed in the rest of this article concern two-thirds of all the dynamic store instances that we have observed. Second, if we choose a benchmark at random, about half the occurrences of store operations seen during its execution tend to be invisible.

\section{CHARACTERIZATION}

In this section, we analyze the impact of different features on the occurrence of silent stores. This study shall be performed on 39,374 stores found in 219 benchmarks. We have removed the three largest benchmarks from our collection (all from SPEC CPU2006: gcc, perlbench, and gobmk), which, together, gives us 50,157 stores. We removed these benchmarks to avoid letting a few programs influence our results: without them, no program accounts alone for more than one-fourth of the stores that we analyze. Nevertheless, our conclusions remain the same, once we consider these three programs. We have made publicly available an extra version of Figure 10 with our full collection of 89,531 stores. These two tables are remarkably similar.

Figure 3 shows that a few features encode enough information to indicate high or low probability of silentness. For instance, Ozr, a feature that characterizes stores that deposit the constant zero in memory, usually indicates silentness. On the other hand, Oic, which characterizes stores whose value is produced by an increment operation, e.g., $\star p=v^{++}$, is a strong indicator of nonsilent behavior. Figure 7(a) shows how the proportion of these two features vary among stores, as we vary the threshold of silentness that we consider. Features Dzr and Din also show an interesting variation pattern, which Figure 7(b) outlines. The former feature characterizes stores whose value depends, directly or indirectly, on the constant zero (of any type). The latter characterizes stores whose value depends on integer constants other than zero. Whereas the former feature is a stronger indication of silentness, the latter indicates the opposite behavior.

Nevertheless, the true power of our features comes out of their combination. To fundament this statement, we have used the a priori algorithm (Agrawal and Srikant 1994) to mine associations among features in our dataset. Figures 8 and 9 highlight a few patterns that we deemed interesting. Each lattice shows the probability of observing a silent store, given a combination of different features. For instance, Figures 8(a) reveals that stores that present the features Pst (store into a field of C-like struct) and Smn (store in the main function) are silent $24 \%$ of the time. ${ }^{1}$ If we add Ozr into this mix, we observe that stores are silent, on average, $79 \%$ of the time. However, this combination of features characterizes only 24 instances of instructions found in our benchmarks. Finally, if we consider also Scm (the store is compulsory within the function where it is located),

\footnotetext{
${ }^{1}$ A note on methodology: in this analysis we report the geometric mean of silentness among the different store instructions that share common features. Thus, the dynamic number of executions plays a small role in the final averages that we use.
} 


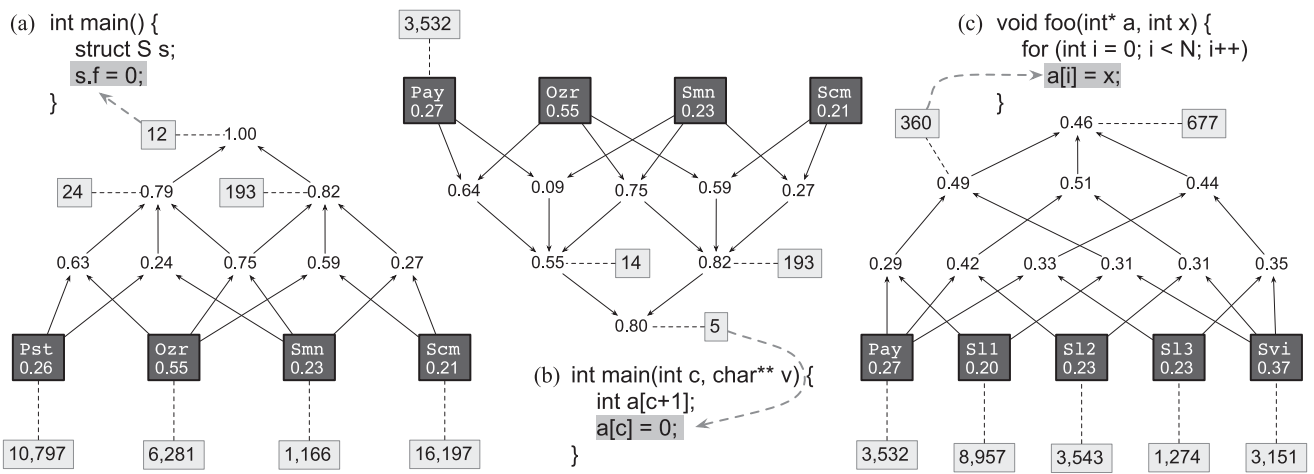

Fig. 8. Three different ways to combine probabilities of silentness. Light gray boxes show the number of static instances of stores sharing a given combination of features. For each lattice we show a program. Dark gray boxes in programs show stores characterized by the feature combination on the top of the lattice.

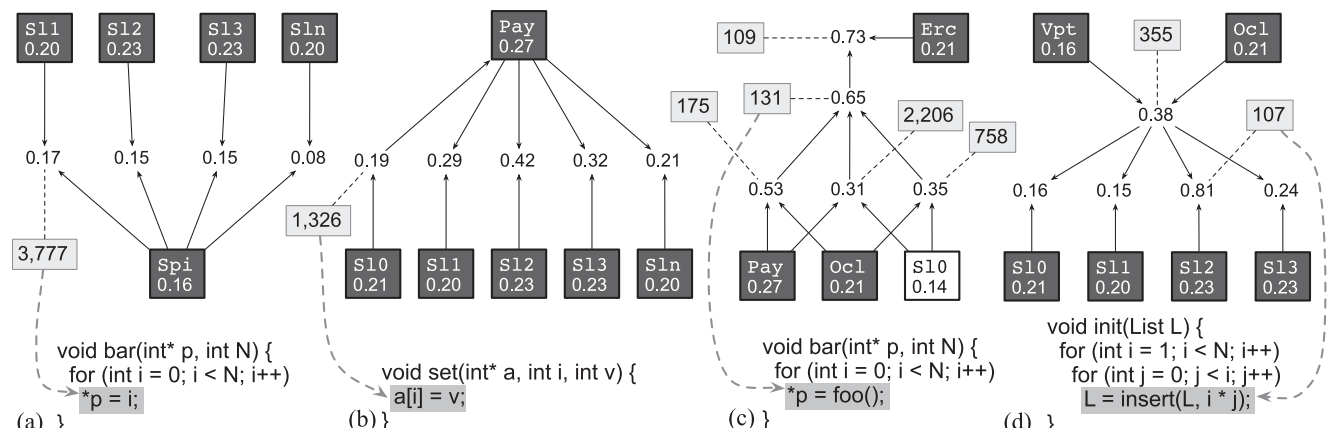

Fig. 9. Probability of silentness for different groups of features. Gray boxes show number of static instances for particular combinations. We show examples of programs for combinations marked with a dark gray box.

then we observe that stores are silent all the time. In this case, only 12 static instances share the four features. Notice that it is relatively easy to produce programs in which instructions sharing these four features are not silent. An example is the second store in "main () \{struct S s; s.f = 1; s.f = $0\}$ ". What our experiment shows is that, at least in the programs that we have, such patterns do not occur-a comprehensible fact, given that rational programmers are unlikely to find any usage for our concocted example. Figures 8(b) and 8(c) show other situations that, intuitively, tend to lead to silentness: compulsory stores of zeros in arrays within the main function, and stores of invariant values into arrays within loops, respectively.

Figure 9 shows other combinations of features that we judged interesting. In the first lattice, we observe that the nesting depth of loops does not seem to impact the silentness of stores targeting invariant pointers. In the second, we see that stores into arrays tend to be more silent as we increase the nesting depth of the surrounding loops, up to two levels of nesting. The initial increase was expected, as by augmenting the depth of loops, we tend to observe more data reuse, i.e., the chance that we revisit the same memory address multiple times. Figures 9(c) and 9(d) highlight facts that we did not, in principle, expect. Figure 9(c) shows that stores into arrays of values produced by functions inside loops tend to be very silent: $65 \%$ across 127 static instances. If such stores are produced by loops controlled by affine induction variables, then this proportion grows further: 73\%. Figure 9(d) indicates that stores of addresses returned by functions are very silent in doubly 

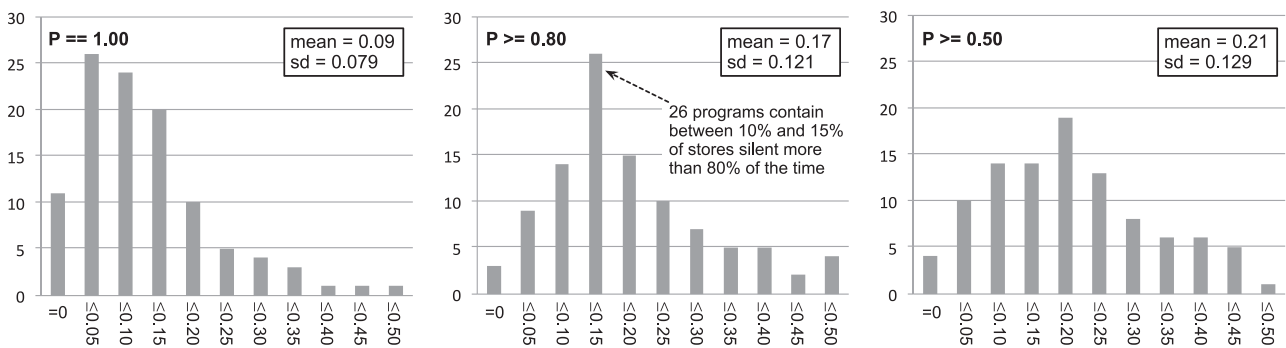

Fig. 10. Distribution of different probabilities of silentness among benchmarks. The $x$-axis shows the proportion of occurrences of static instances with a certain threshold of silentness $P$. The $y$-axis shows the number of benchmarks. The histograms use the 100 largest benchmarks that we have.

nested loops. We deemed this fact unanticipated enough to deserve some investigation. In this case, we believe that such a situation is caused by an anomaly-82 static instances occur in the benchmark consumer-lame/13bitstream.c, and they are $100 \%$ silent. We have associated this behavior with a performance bug: these stores consist of values returned by the same function, and they are never read after been written:

$\star$ pph $=$ BF_addEntry $(* p p h$, scalefac[gr] $[\mathrm{ch}] .1[\mathrm{sfb}]$, slen1) ;

We believe that this performance bug is due to code reuse: the function of interest returns a value, which ends up stored in variable pph, which is dead. Function BF_addEntry is being invoked for the side effects that it produces, not for the value that it returns.

\subsection{On the Frequency of Silentness}

Figure 10 shows the number of occurrences of silent stores with different probabilities per benchmark. To build these histograms, we have used the 100 benchmarks with the largest number of static instances of stores that were executed during profiling. Using this metric, the largest benchmark is perlbench, with 4,539 stores executed, followed by tramp3d-v4, with 3,087 . The smallest benchmark is matmul_f64_4x4, with 19 stores. The figure suggests that the distribution of silentness across benchmarks follows a normal distribution. We provide the population parametersmean and standard deviation-for different thresholds of silentness, e.g., the $P$ in Definition 2.1. The number of occurrences of store operations meeting a certain threshold of silentness increases with the increase of this threshold. We did not show the histogram for $P=0.00$, but, in this case, we have mean equal to 0.616 and standard deviation equal to 0.134 . That is to say that, in the 100 benchmarks used in Figure 10,61.6\% of the stores were never silent; i.e., they were always noisy.

Figure 11 shows a profile of silentness. To build this chart, we have grouped stores according to their feature vectors. Each point in the x-axis of the figure represents a feature vector that characterizes at least 10 different static store instances. This number, 10, is arbitrary: we chose it because, otherwise, we would have too many feature vectors to consider. Furthermore, removing vectors that are not frequent reduces the chance that our results become marred by data that is unlikely to appear in practice. The y-axis gives us the fraction of static instances, within a feature vector, that were silent above a given threshold (we consider $80 \%$ and $50 \%$ of silentness).

Figure 11 shows that feature vectors are relatively sparse. In total, we found 8,036 different feature vectors characterizing our 39,374 store instructions. If we separate only the groups that have 10 or more instances, we obtain 593 different feature vectors. The universe of possible feature vectors is very large: $U=6 \times 10 \times 17 \times 17 \times 20 \times 2^{9} \times 2^{16} \times 2^{32}$. This number comes from the fact 


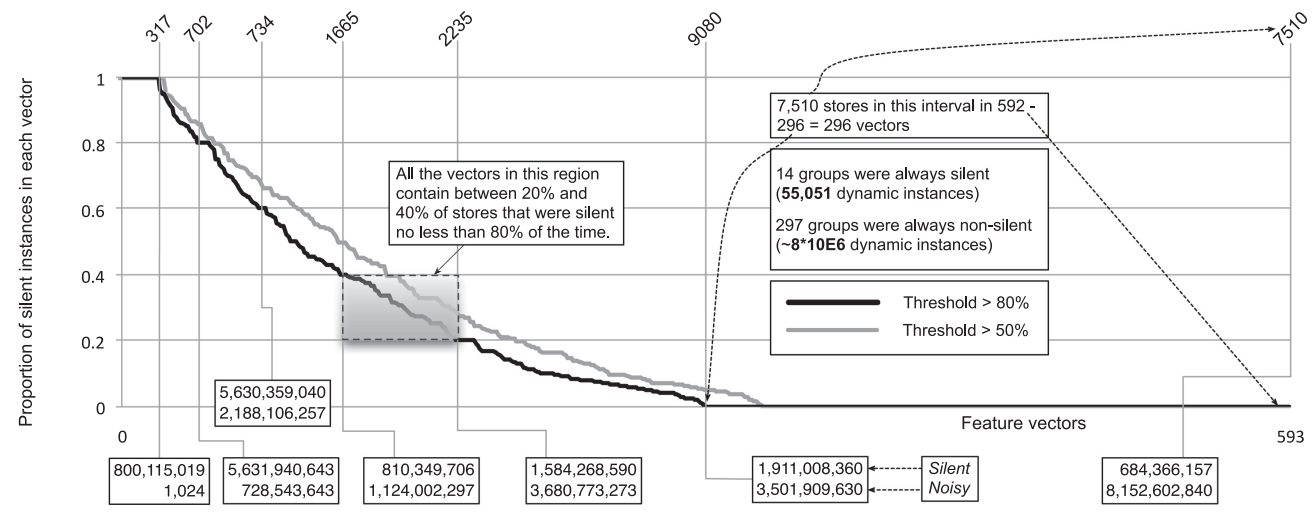

Fig. 11. The profile of silentness. Each point in the $x$-axis represents a different vector of features that appears in at least 10 static instances. The $y$-axis is the proportion of static instances, within each vector, that were silent $80 \%$ of the time, or $50 \%$ of the time. Numbers on the top show quantity of static instances in given ranges of silentness. Boxes on the bottom indicate quantity of dynamic instances (top of the box: silent stores; bottom: noisy stores) observed during profiling.

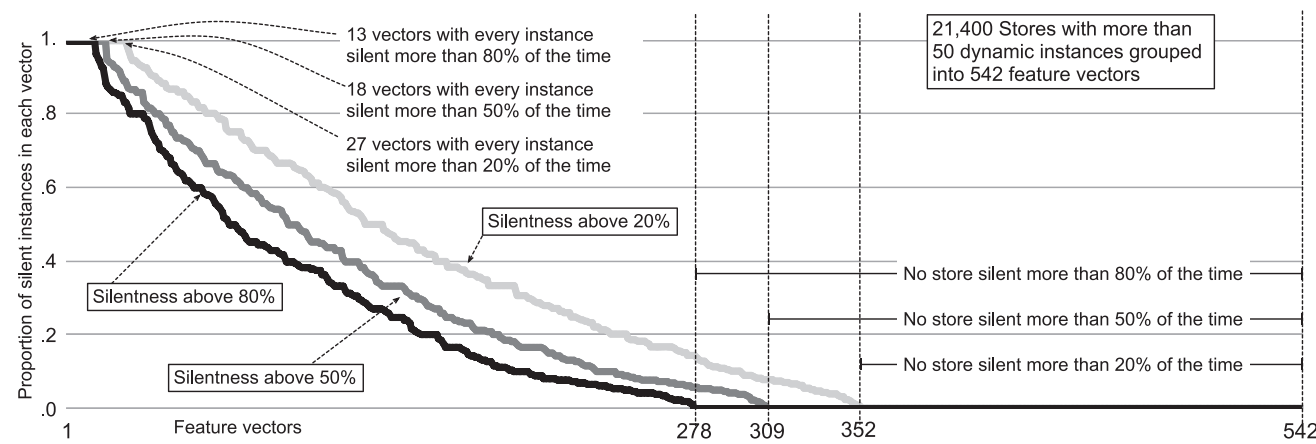

Fig. 12. The profile of silentness for "hot" stores, i.e., stores with at least 50 dynamic instances. We have observed 542 vectors with 10 or more static instances that run more than 50 times. As in Figure 11, each point in the $\mathrm{x}$-axis represents a different vector of features that appears in at least 10 static instances. The $y$-axis is the proportion of static instances, within each vector, that were silent $80 \%, 50 \%$, or $20 \%$ of the time. Numbers on the top show quantity of static instances in given ranges of silentness.

that five categories of features (with 6,10,17, or 20 elements) must have exactly one representative element in the vector, whereas the other three (ValueDeps, LabelLoc, and PointerStride) can have multiple representatives. The fact that features are concentrated into a negligible fraction of the universe of vectors, e.g., 8,036/U, indicates regularity among real-world programs.

Another interesting observation is that "silent stores are very silent, and noisy stores are very noisy." To explain the meaning of this statement, Figure 11 contains two thresholds of silentness: vectors are grouped according to the fraction of instructions that are silent $80 \%$ or $50 \%$ of the time. The two lines are similar. This characteristic of our data is not due to most of the stores executing only once during profiling. The most-often-seen number of executions of stores is indeed one: out of the 39,374 stores that we have analyzed, 6,001 run only once. However, 24,209 instructions run 50 or more times. If we create a histogram with only those "hot" stores, then we obtain a picture similar to Figure 11. Figure 12 shows this result. Because we only consider hot stores in this new 

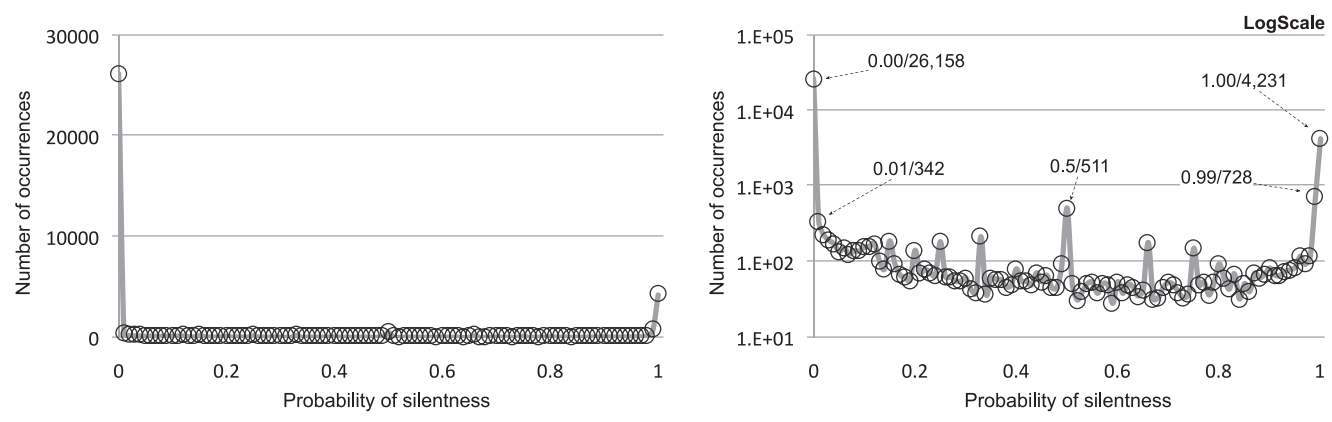

Fig. 13. Proportion of silent stores observed at runtime. Both figures show the same information. The chart on the right uses logarithmic scale, to accentuate key values.

experiment, we obtain 542 feature vectors, slightly less than the 593 vectors seen in Figure 11 . Nevertheless, the relation between different levels of silentness remains very similar.

To make this result-silent stores are very silent, noisy stores are very noisy-even more evident, Figure 13 shows a histogram of the number of static stores, grouped by the probability of silentness observed via profiling. The $\mathrm{x}$-axis shows the probability of silentness (101 buckets), and the y-axis reports the number of static store instances. The area under the curve is the number of stores observed: 39,374. The pair prob/num indicates that num stores were silent with probability prob. The U-shaped histogram indicates that most of the stores are either always or never silent. To ease visualization, we show this histogram also in logarithmic scale.

\section{PREDICTION}

This section explores several ways to address the problem stated in Definition 2.1. Different dynamic instances of the same store operation may behave differently: being silent at some executions and noisy at others. Therefore, prediction is meaningful given a certain probability. Hence, we are interested in guessing which stores are silent with some probability $P$, as seen in Definition 2.1.

\subsection{Precision, Recall, and Accuracy}

Figure 13 suggests that our dataset is skewed. Most of the dynamic store instances are noisy: silent store operations account for less than $20 \%$ of all the writes in memory. Therefore, if we simply predict stores as noisy, we are going to be right often and, thus, highly accurate. Nevertheless, by assuming that every store is noisy, we will never predict correctly the behavior of stores that tend to be silent. Therefore, accuracy - the ratio between correct predictions and total predictions-is not a good metric for our dataset. A better metric is the notion of F1-score, which we revisit below:

Definition 4.1 (Prediction Metrics). Given the notions of true positives (TP), true negatives $(T N)$, false positives $(F P)$, and false negatives $(F N)$ seen in Definition 2.1, we define the following metrics:

$$
\begin{aligned}
\text { Precision } & =T P /(T P+F P) \\
\text { Recall } & =T P /(T P+F N) \\
\text { F1-Score } & =2 \times \text { Precision } \times \text { Recall } /(\text { Precision }+ \text { Recall }) \\
\text { Accuracy } & =(T P+T N) /(T P+T N+F P+F N)
\end{aligned}
$$

"Precision" indicates, out of the stores predicted as silent, how many were observed to be silent. "Recall" tells how many actually silent stores were predicted as such. A predictor that guesses every store as noisy would have zero recall, and an undefined precision, due to division by zero. 


\begin{tabular}{|c|c|c|c|c|c|c|}
\hline \multirow[b]{2}{*}{ store 1} & \multicolumn{2}{|c|}{$\begin{array}{ll}a & \mathbf{b}\end{array}$} & \multicolumn{2}{|c|}{ c d } & \multicolumn{2}{|c|}{ ss ns } \\
\hline & 1 & 0 & 1 & 0 & 3 & 5 \\
\hline store 2 & 1 & 0 & 0 & 1 & 1 & 6 \\
\hline store 3 & 0 & 1 & 1 & 0 & 8 & 0 \\
\hline store 4 & 1 & 0 & 1 & 1 & 3 & 9 \\
\hline store 5 & 0 & 1 & 1 & 1 & 3 & 2 \\
\hline store 6 & 1 & 0 & 0 & 0 & 1 & 8 \\
\hline store 7 & 1 & 0 & 0 & 1 & 2 & 4 \\
\hline store 8 & 0 & 1 & 0 & 0 & 3 & 8 \\
\hline store 9 & 0 & 1 & 1 & 1 & 8 & 1 \\
\hline
\end{tabular}

Fig. 14. Example of dataset.

\subsection{The Oracle: An Upper Bound on Accuracy}

A static predictor can only use the vector of features associated with a store operation when taking a decision concerning that store. Thus, different static instances characterized by the same vector should be predicted in the same way. Figures 11 and 12 show that our benchmark suite contains vectors associated with static instances that behave differently. The light gray box in Figure 11 shows examples of such stores. Each vector in that region contains at least 10 different static store instances, a number of which (between $20 \%$ and $40 \%$ ) were silent more than $80 \%$ of the time, while others were not. Therefore, any static predictor is bound to make mistakes in part of the elements of each of these vectors, because prediction must be uniform for all the elements within the same vector. Better predictors make fewer such mistakes, up to what can be accomplished by an oracle. An oracle is an ideal predictor, defined as follows:

Definition 4.2 (Oracle). Given an instance $(I, l, P)$ of the static prediction problem (see Definition 2.1), an oracle produces the following output:

- Let $V$ be the vector of features that characterize $t$.

- Let $s s$ be the number of stores in $I$ characterized by $V$, which have been observed to be silent with probability greater than or equal $P$.

- Let $n s$ be the number of stores in $I$, also characterized by $V$, which have been observed to be silent with probability less than $P$.

-If $s s \geq n s$, then predict $\iota$ as "silent"; else predict $\iota$ as "noisy."

The algorithm seen in Definition 4.2 is optimal, because all the stores grouped in the same feature vector must be predicted in the same way. There are only two possible predictions (silent or noisy), and the oracle is correct for at least half of them.

As an example, let us see how the oracle fares on the dataset seen in Figure 14. If we consider a probability of silentness larger than 0.5 , i.e., $P>0.5$, then we have three true positives and six true negatives. No false positives or false negatives are observed. This result gives us maximum precision and recall and hence, an optimal F1-score of 1.00. If we consider $P>0.8$, then we have one false positive due to the vector $(0,1,1,1)$. Stores 5 and 9 are characterized by this vector. The first vector is silent $3 /(2+3)$ times; hence, this instance does not meet the threshold of 0.8 , and is considered noisy (we are interested in $P>0.8$ ). On the other hand, instance 9 surpasses the threshold, and is considered silent, because $8 /(1+8)>0.8$. The oracle marks both instances as silent, thus yielding one false positive. Finally, if we consider $P==1.0$, then the oracle predicts every store as noisy, hence having only true negatives, e.g., nine in total. In this extreme case, even though accuracy is maximum, e.g., $100 \%$, we cannot define the F1-score, as the number of true positives is zero. 


\begin{tabular}{|c|c|c|c|c|c|c|c|c|}
\hline & & & & & & & \multicolumn{2}{|c|}{$P=0.50$} \\
\hline & TP & TN & FP & $\mathbf{F N}$ & Prec & $\operatorname{Rec}$ & F1 & Accy \\
\hline Oracle & 3 & 6 & 0 & 0 & 1.00 & 1.00 & 1.00 & 1.00 \\
\hline \multirow{3}{*}{$\begin{array}{l}\text { Always-silent } \\
\text { Always-noisy }\end{array}$} & 3 & 0 & 6 & 0 & 0.33 & 1.00 & 0.50 & 0.33 \\
\hline & 0 & 6 & 0 & 3 & Und & 0.00 & Und & 0.67 \\
\hline & 3 & 2 & 4 & 0 & 0.43 & 1.00 & 0.60 & 0.56 \\
\hline
\end{tabular}

\begin{tabular}{|c|c|c|c|c|c|c|c|c|}
\hline & \multicolumn{8}{|c|}{$P=0.80$} \\
\hline & TP & TN & FP & FN & Prec & Rec & F1 & Accy \\
\hline \multirow{4}{*}{$\begin{array}{r}\text { Oracle } \\
\text { Always-silent } \\
\text { Always-noisy } \\
\text { Random }\end{array}$} & 2 & 6 & 1 & 0 & 0.67 & 1.00 & 0.80 & 0.89 \\
\hline & 2 & 0 & 7 & 0 & 0.22 & 1.00 & 0.36 & 0.22 \\
\hline & 0 & 7 & 0 & 2 & Und & 0.00 & Und & 0.78 \\
\hline & 0 & 6 & 1 & 2 & 0.00 & 0.00 & Und & 0.67 \\
\hline
\end{tabular}

Fig. 15. Results produced by the predictors seen in Sections 4.2 and 4.3 on the dataset in Figure 14.

\subsection{The Null Hypothesis}

In the context of this work, we consider a Null Hypothesis the statement that the behavior of dynamic store instances is not related to the feature vectors that characterize the corresponding static store instances. To disprove the null hypothesis, we consider three trivial predictors and show how their F1-scores compare with the oracle and with the heuristics that we describe in Section 4.4. The three trivial predictors that we consider are defined as follows:

Definition 4.3 (Trivial Predictor). A trivial predictor is an $O(1)$ solution to the static prediction of silent stores that does not consider program features. We consider the following trivial predictors:

- Always-noisy: assumes that every instance of the silent store prediction problem is noisy

- Always-silent: predicts every instance of the problem as silent

- Random: given an instance $(I, l, P)$ of the prediction problem, draws a random variable $X$ from a uniform distribution in $[0,1.0]$ and predicts the instance as silent if $X \leq P$

Even though trivial, the predictors listed in Definition 4.3 give us useful information. For instance, the accuracy of the always-noisy predictor corresponds to the proportion of noisy dynamic store instances, whereas the accuracy of the always-silent predictor tells us the proportion of silent store operations in our dataset. The former tends to be very accurate, given that most of the dynamic instances of stores are noisy. However, its recall is always zero, because it will never output a true positive, and its precision is undefined, due to a division by zero. The always-silent predictor, in turn, has maximum recall, provided that the dataset contains at least one silent store. Nevertheless, its F1-score will be low, given that it tends to present low precision.

Example 4.4 (Trivial Predictors). Figure 15 shows how the trivial predictors perform on the data in Figure 14. Predictions produced by oracle, always-silent, and always-noisy are always deterministic. The result of the random predictor varies from one execution to another, and its accuracy increases with $P$. For $P=0.80$, the oracle mispredicts one result, due to the reasons discussed in Section 4.2.

Figure 16 (left) shows how the three predictors of Definition 4.3 fare, when compared against the oracle of Definition 4.2, in terms of F1-score and accuracy. We analyze how each predictor performs when we consider three different probabilities of silentness: $0.5,0.8$, and 1.0 . The probability of 0.8 , for example, indicates an instance of the prediction problem whose goal is to determine which store instructions are silent more than $80 \%$ of the time. To produce Figure 16, we consider only $10 \%$ of the dataset, i.e., 5,906 instructions in a so-called test set. ${ }^{2}$ When splitting the data, we ensured that stores within the same benchmark remained grouped together: benchmarks were sorted lexicographically and separated into three sets: training (27,562 stores), validation (5,906 stores), and test (5,906 stores). In this way, we preserve the regularity that stores tend to share within the same benchmark-a fact that we shall revisit in Section 4.4. As Figure 16 indicates, the F1-score of

\footnotetext{
${ }^{2}$ This restriction is necessary to let us carry out uniform comparisons between different predictors. The predictors that we shall see in Section 4.4 require us to separate part of the dataset for training, validation, and testing purposes.
} 

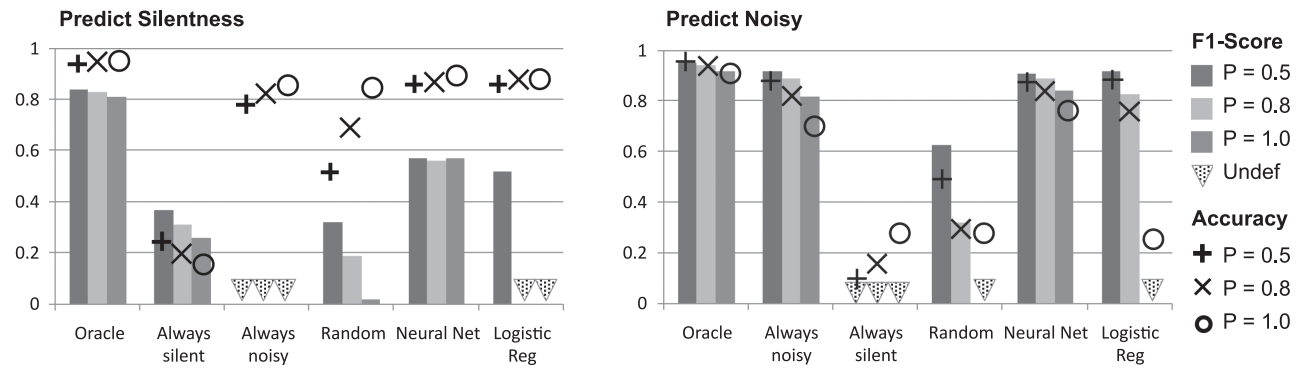

Fig. 16. F1-score produced by different predictors on $10 \%$ of the dataset. Undef denotes division by zero, due to either precision or recall being zero. $P=X$ denotes an instance of the problem whose goal is to predict stores that are silent (or noisy) with probability $P$. The higher the bar, the better is the predictor.
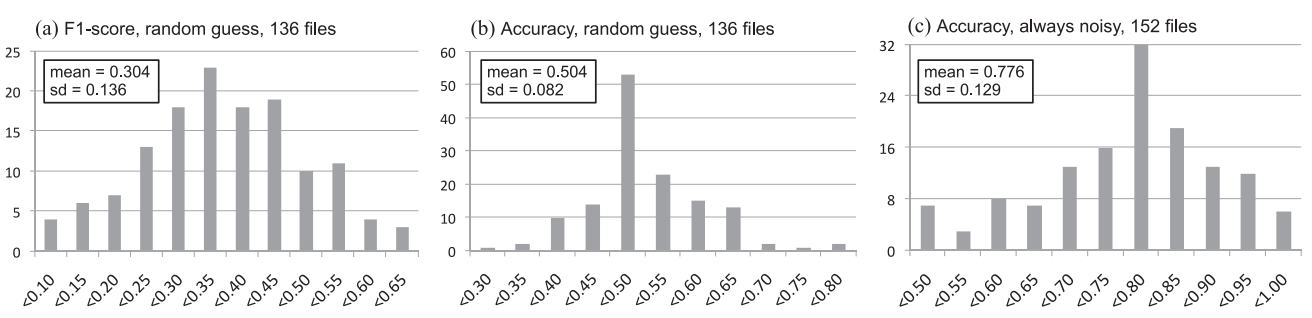

Fig. 17. Distribution of F1-score and accuracy produced by different trivial predictors, considering $P=0.5$.

any trivial predictor is never above half that observed in the oracle. In particular, if we raise the threshold of silentness to 1.0, then the F1-score of the random predictor is barely above zero.

The chart on the right side of Figure 16 shows the same comparison as the chart on the left, except that in this case we have inverted the goal of the predictor. Thus, instead of predicting silent stores, we are trying to predict noisy instances. In this case, the notions of true and false positives (or negatives) are inverted. The purpose of this figure is to illustrate the impact of skewing in our results. In this new scenario, the high rate of noisy stores causes all the trivial predictors to approximate the oracle in terms of accuracy, precision, and recall, hence obtaining similar F1-scores.

Refuting the Null Hypothesis. We close this section by analyzing the following question: when is a predictor doing more than the trivial predictors do? To answer this question, we focus on two trivial predictors: random guess and always noisy. The F1-score of the former and the accuracy of both tend to follow a normal distribution. To support this statement, we have computed population parameters for our individual benchmarks. Figure 17 provides histograms of these three populations. To produce these histograms, we chose only benchmarks with at least 10 different store instructions that were executed during profiling. Additionally, for two charts (Figures 17(a) and 17(b)), we filtered out benchmarks with undefined F1-scores. The F1-score is undefined when precision or recall are zero. This lot gave us 152 benchmarks. Additionally, to produce Figures 17(a) and 17(b), we removed benchmarks that did not present any dynamic instance of silent stores with probability of 50\%. This filtering reduced the number of benchmarks down to 136 .

From these samples, we used Student's Test (Hill 1970) to find intervals of confidence within which we should expect to find similar results (F1-score and accuracy) produced from different samples. For instance, random guessing over our 136 samples gives us an F1-score whose confidence interval (at 95\%) lies between 0.168 and 0.44 . Thus, if we obtain an F1-score above 0.44, then we consider it statistically significant. The accuracy of the random guess covers the interval between 0.422 and 0.586 with confidence of $95 \%$. Finally, the accuracy of the "always noisy" 
predictor is expected to fall between 0.647 and 0.905 , also with confidence of $95 \%$. To give the reader some perspective on this number, the chance that random guessing gives us an F1-score of 0.40 , when given a collection with the same size of 136 samples, is inferior to $1.16 \mathrm{e}-08$. The predictors of Section 4.4, which are based on regression models, can go much beyond that mark.

\subsection{Statistical Regression}

If a predictor is allowed to observe the behavior of some store operations, then it can use the result of this observation to estimate the probability that unseen stores will be silent or noisy. This modus operandi is known as supervised learning. To train a predictor, part of the available data is split into training and test sets. In this article, we consider two models: logistic regression and a neural network based on back-propagation. Logistic regression is an overly simplistic model; however, it gives us the necessary equipment to explain the intuition behind regression in general.

4.4.1 Logistic Regression. A feature vector with $n$ features is an $n$-dimensional vector of Booleans. We have that a training dataset $D_{t r n}=\left(\iota^{\prime}, s s, n s\right)^{m}$ is a list with $m$ entries. Each entry contains a static store instance $\iota^{\prime}$, a number of occurrences of silent instances $s s$, and a number of occurrences of noisy instances $n s$, observed via profiling. From such a training set, we can draw $m$ feature vectors $V$. The logistic regression problem (Cox 1958) consists of finding an $n$-dimensional vector $\Theta$, so that the product $V \Theta$ gives us a way to predict the behavior of stores characterized by $V$ :

Definition 4.5 (Logistic Regression). Given an instance $(I, l, P)$ of the static prediction problem:

- Let $V_{\iota} \in\{0,1\}^{n}$ be the $n$-dimensional binary vector that characterizes instruction $\iota$.

- Let $\Theta \in \mathbb{R}^{n}$ be an $n$-dimensional vector.

- Let the sigmoid function be defined as $\sigma(V \Theta)=\frac{1}{1+e^{-V \Theta}}$.

- if $\sigma(V \Theta) \geq P$ then Predict $\iota$ as silent else predict $\iota$ as noisy.

From Definition 4.5, the problem of constructing a predictor based on logistic regression consists of finding a vector $\Theta$. This vector must maximize the quantity of correct predictions on the training set. The underlying assumption is that a $\Theta$ that approximates the behavior of the training set is likely also to yield reasonable results on the test set. There exist efficient techniques to find a suitable vector $\Theta$. The method of the gradient descent is the most well-known technique (Cauchy 1847). Thus, henceforth, we shall assume that $\Theta$ can be efficiently approximated for any training set.

Example 4.6 (Solving Logistic Regression). Figure 18 shows how logistic regression finds the vector $\Theta$ for a training set with nine entries and $P=0.5$. In this example, gradient descent is able to find a vector $\Theta=(-0.48,-0.44,0.44,0.49,0.02)$. This algorithm is not deterministic: other $\Theta s$ could also have been found. Using the algorithm "if $\sigma(V \Theta) \geq 0.5$ (e.g., $P=0.5$ ) then silent else noisy," we obtain a perfect score of six true negatives, and three true positives on the training set.

Once we have a vector $\Theta$, we can use it to predict the behavior of store instructions that were not used in the process of building it. In other words, the output of the training phase of the logistic regression is this vector $\Theta$, which later gives us the means to carry out predictions. Thus, a predictor based on logistic regression receives two inputs: the target program containing the stores that must be analyzed, plus the vector $\Theta$ built during the training phase. Our predictor uses the feature mining machinery described in Section 2.2.2 to produce a vector for each store in the target program. Given such vectors, we resort to Definition 4.5 to infer the behavior of the corresponding store operations.

Example 4.7 (Applying Logistic Regression on the Test Set). Figure 19 shows the result of applying the vector $\Theta$ found in Example 4.6 on a test set with four entries. The predictor correctly guesses 


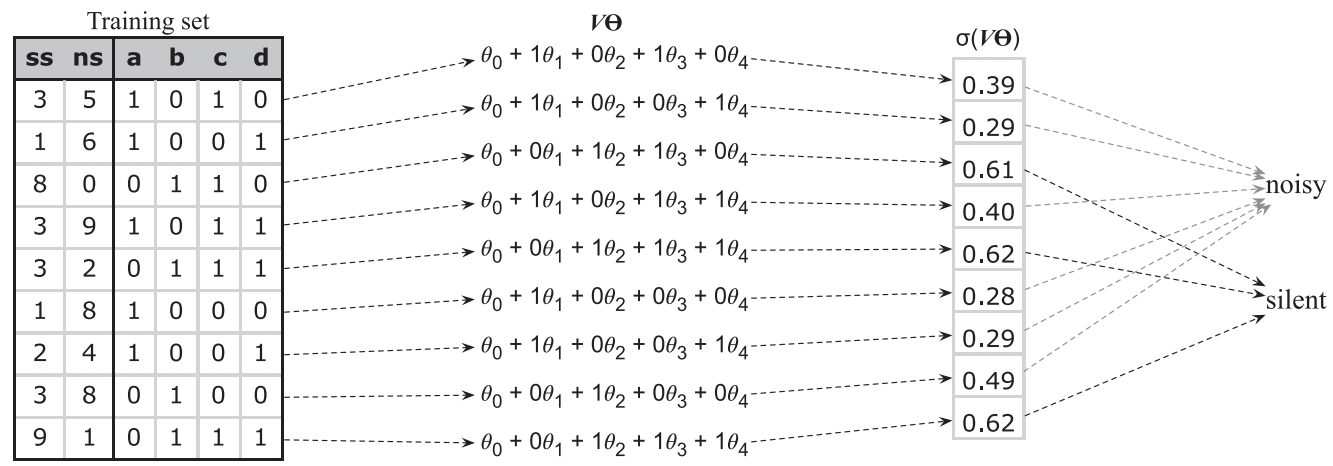

Fig. 18. Results produced by logistic regression on the training set. In this example, gradient descent finds the solution $\theta_{0}=-0.48, \theta_{1}=-0.44, \theta_{2}=0.44, \theta_{3}=0.49$, and $\theta_{4}=0.02$. All the arrows toward the "noisy" label denote true negatives, and all the arrows towards the "silent" label denote true positives.

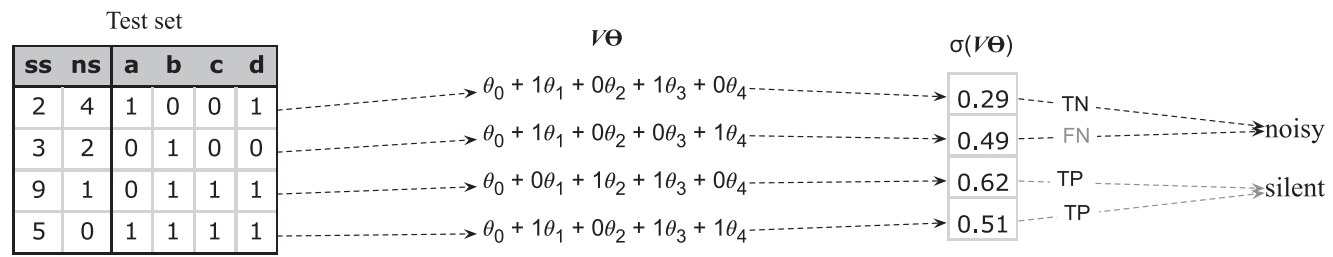

Fig. 19. Results produced by logistic regression on the test set, using the same $\Theta$ vector found in Figure 18 .

the behavior of the vector $(1,1,1,1)$, which was not present in the training set. Successful prediction is possible because this vector is sufficiently similar to the vectors in the training set that characterize silent occurrences of stores, namely, $(1,0,1,1)$ and $(0,1,1,1)$. However, our predictor fails to correctly guess the behavior of $(0,1,0,0)$. The store characterized by this vector is predicted as noisy, although three out of five dynamic instances of it are silent in practice.

Figure 16 (left) compares logistic regression against other predictors. Its F1-score is almost twice as high as the random predictor, for $P=0.5$. However, when considering higher probabilities, e.g., $P=0.8$ and $P=1.0$, our implementation of logistic regression tends to predict all the stores as noisy, hence approximating the behavior of the "always-noisy" predictor. Logistic regression uses a convex objective function $\sigma(V \Theta)$. Thus, gradient descent will yield an optimal global solution to it, given enough time to converge. Unfortunately, there is no indication that the universe of feature vectors can be correctly separated into silent and noisy groups by a convex surface. The results in Figure 16 seem to imply that such separation is not possible. Thus, any solution to our logistic regression model is likely to be a rough approximation of the actual behavior of store instructions.

4.4.2 Neural Network. The subpar performance of logistic regression is due to its inability to relate different features. For instance, if we have two features, $f_{1}$ and $f_{2}$, the conclusions of logistic regression come from the linear combination $\theta_{0}+\theta_{1} f_{1}+\theta_{2} f_{2}$. The product $f_{1} f_{2}$ is immaterial in this function. In this section, we expand the notion of statistical regression to discuss a model that can accommodate that product, among other nonlinear relations. This model, based on a backpropagation neural network, is defined as follows: 


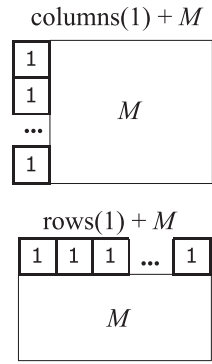

(a)

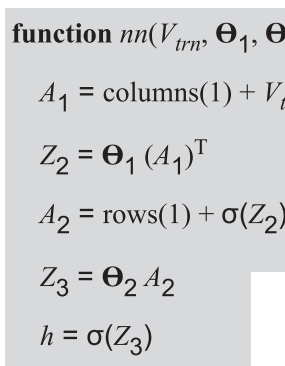

(b)

\begin{tabular}{|c|c|c|c|c|c|}
\hline \multirow[t]{2}{*}{$\boldsymbol{\theta}_{1}$} & -0.76 & -1.54 & 0.93 & 0.92 & -0.06 \\
\hline & -0.81 & 0.67 & 0.33 & -1.07 & -0.87 \\
\hline & 0.54 & 0.97 & 0.64 & -0.29 & -0.39 \\
\hline & -0.11 & 0.63 & 0.22 & -1.44 & 0.01 \\
\hline & 0.76 & 0.12 & 0.49 & 0.21 & -0.10 \\
\hline & 0.64 & -0.82 & 0.18 & 0.43 & -1.02 \\
\hline & 0.03 & -0.18 & -0.70 & -0.64 & -0.15 \\
\hline & -0.89 & 0.37 & 0.94 & -0.64 & -0.37 \\
\hline & $-0.9-c$ & $\begin{array}{ll}.5 & -1.7\end{array}$ & 1.0 & $0.4-1$ & 0.0 \\
\hline
\end{tabular}

(c)

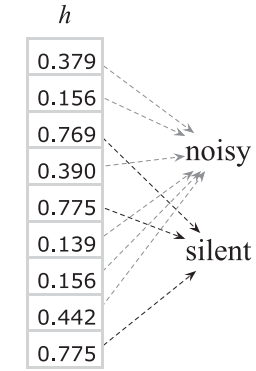

(d)

Fig. 20. After training the neural network, we obtain two matrices: $\Theta_{1}$ and $\Theta_{2}$, which lets us compute the vector $h$. (a) Notation used to denote operations that augment matrices. (b) The expression used to compute predictions. $A^{T}$ is the transpose of matrix $A$. (c) Matrices $\Theta_{1}$ and $\Theta_{2}$ computed for the training set in Figure 18, for $P=0.5$. Vector $h ; h[i] \geq P$ indicates that $i^{t h}$ store is silent.

Definition 4.8 (Single-Layer Perceptron). Given instance $(I, \iota, P)$ of the static prediction problem:

- Let $V_{\iota} \in\{0,1\}^{n}$ be the $n$-dimensional feature vector that characterizes instruction $\iota$.

- Let $\Theta_{1} \in \mathbb{R}^{n+1} \times \mathbb{R}^{L}$ and $\Theta_{2} \in 1 \times \mathbb{R}^{L+1}$ be matrices of real numbers.

$-L$ is called the size of the hidden layer.

- Let the $n n$ function be defined as in Figure 20(b), where $\sigma(x)=\frac{1}{1+e^{-x}}$.

- if $h(V) \geq P$ then Predict $\iota$ as silent else predict $\iota$ as noisy.

In this article, we use a feed-forward single-layer neural network (also known as Single-Layer Perceptron), which is defined by the matrices $\Theta_{1}$ and $\Theta_{2}$ of Definition 4.8. More layers would add more matrices into our definition. This neural network has one input layer, with one neuron per feature in our model; a hidden layer, with $L$ neurons; and one output layer, with a single node $h$. Metaphorically, to use the network, we assign the value of each feature to the corresponding input neuron. The value of the output node is determined by function $n n$, which appears in Figure 20.

Just like in the case of logistic regression, seen in Section 4.4.1, to build a predictor based on the neural network, we need to find good matrices $\Theta_{1}$ and $\Theta_{2}$. To this end, we rely on the training set. We perform multiple applications of function $n n$ onto the vectors that we derive from the training set, using gradient descent to approximate candidate matrices. We say that an approximation is good if it yields values of $h$ that maximize the amount of correct predictions on the training set.

Example 4.9 (Training of Neural Network). Figure 20(c) shows the matrices $\Theta_{1}$ and $\Theta_{2}$ that some rounds of gradient descent produce for the training set seen in Figure 18. These matrices yield a perfect fit on this training set, as Figure 20(d) shows. The value of $h$ computed for each vector in the training set gives us an exact prediction of the behavior of that vector, for $P \geq 0.5$.

After finding concrete values for matrices $\Theta_{1}$ and $\Theta_{2}$, we use them to build a predictor, like we did for logistic regression. This new predictor receives the program to be analyzed and the two matrices. It mines features for each store in that program and uses the $n n$ function to predict the outcome of that store. We emphasize that prediction is a fast process, which consists of the time of mining features plus the time to run equation $n n$. Feature mining is linear on the program size, as Figure 5 indicates. The runtime of function $n n$ depends only on the number of features per store; hence, it is constant for a given choice of features. Example 4.10 illustrates this process.

Example 4.10 (Applying the Neural Network on the Test Set). Figure 21 shows how our neural network performs, when given the matrices found in Example 4.9, and the test set earlier seen in 


\begin{tabular}{|c|c|c|c|c|c|c|c|c|}
\hline \multicolumn{5}{|c|}{$A_{1}=$ columns $(1)+V$} & \multicolumn{4}{|c|}{$Z_{2}=\boldsymbol{\Theta}_{1}\left(A_{1}\right)^{\mathrm{T}}$} \\
\hline 1 & a & b & c & d & -2.36 & 0.17 & 1.03 & -0.51 \\
\hline 1 & 1 & 0 & 0 & 1 & -1.01 & -0.48 & -2.42 & -1.75 \\
\hline 1 & 0 & 1 & 0 & 0 & 1.12 & 1.18 & 0.50 & 1.47 \\
\hline 1 & 0 & 1 & 1 & 1 & 0.53 & 0.11 & -1.32 & -0.69 \\
\hline \multirow[t]{4}{*}{1} & 1 & 1 & 1 & 1 & 0.78 & 1.25 & 1.36 & 1.48 \\
\hline & & & & & -1.20 & 0.82 & 0.23 & -0.59 \\
\hline & & & & & -0.30 & -0.67 & -1.46 & -1.64 \\
\hline & & & & & -0.89 & 0.05 & -0.96 & -0.59 \\
\hline
\end{tabular}

$A_{2}=\operatorname{rows}(1)+\sigma\left(Z_{2}\right)$
\begin{tabular}{|c|c|c|c|}
\hline 1 & 1 & 1 & 1 \\
\hline 0.09 & 0.54 & 0.74 & 0.38 \\
\hline 0.27 & 0.38 & 0.08 & 0.15 \\
\hline 0.75 & 0.76 & 0.62 & 0.81 \\
\hline 0.63 & 0.53 & 0.21 & 0.33 \\
\hline 0.69 & 0.78 & 0.8 & 0.81 \\
\hline 0.23 & 0.69 & 0.56 & 0.36 \\
\hline 0.43 & 0.34 & 0.19 & 0.16 \\
\hline 0.29 & 0.51 & 0.28 & 0.36 \\
\hline
\end{tabular}

\begin{tabular}{|c|c|c|c|}
\hline \multicolumn{4}{|c|}{$Z_{3}=\boldsymbol{\theta}_{2} A_{2}$} \\
\hline-1.69 & -0.23 & 1.23 & 0.03 \\
\hline \multicolumn{4}{|c|}{$h=\sigma\left(Z_{3}\right)$} \\
\hline 0.16 & \multirow{2}{*}{\multicolumn{3}{|c|}{ … TN …łnoisy }} \\
\hline $0.44^{-}$ & & & \\
\hline 0.77 & \multirow{2}{*}{\multicolumn{3}{|c|}{$\cdots$ TP $\ldots$. ... }} \\
\hline 0.51 & & & \\
\hline
\end{tabular}

Fig. 21. The different matrices produced by function $n n$ (Figure 20(b)), using $\Theta_{1}$ and $\Theta_{2}$ from Figure 20(c).

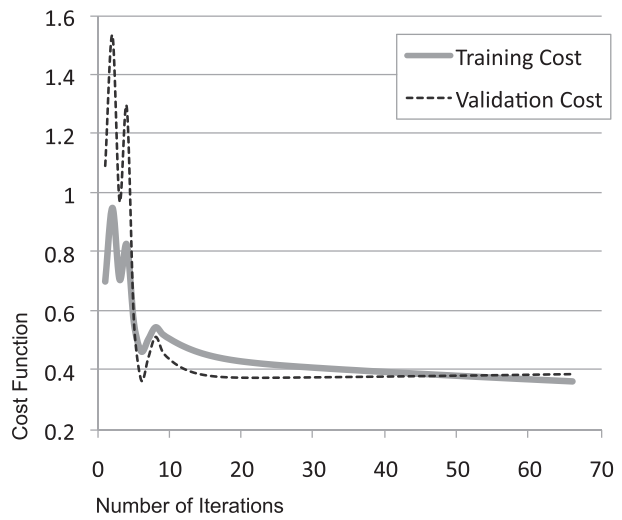

Fig. 22. Convergence of the perceptron model.

Figure 18. We show each one of the intermediate results produced upon running function $n n$ onto this test set. In this example, our predictor is correct three times and misses one instance. As when using logistic regression, we output an incorrect result for $(0,1,0,0)$.

Example 4.10 suggests that the neural network produces results similar to those generated by logistic regression; however, the performance of these two predictors is very different. Figure 16 shows how the single-layer perceptron compares to the other predictors used in this article. Its accuracy is similar to that of other predictors; however, its F1-score is substantially better. We obtain scores of $0.57,0.55$, and 0.56 for $P=0.5, P=0.8$, and $P=1.00$, respectively. This F1-score is about twice as high as that obtained by the other predictors and is about $70 \%$ of the oracle's.

To obtain the results in Figure 16, we have used a hidden layer with twice as many neurons as features, e.g., $L=2|V|$. Other configurations that we have used do not lead to better F1-scores. We have experimented with larger $L s$ and with more hidden layers. More complex networks tend to cause overfitting: our choice of $\Theta$ s fits too well the training set but performs poorly on unseen data. To avoid overfitting, we use $10 \%$ of our data as a validation set. We stop gradient descent once the F1-score starts diverging between the training and validation sets.

Figure 22 illustrates this trend. This chart plots the cost function used by gradient descent on the $y$-axis and the number of iterations of gradient descent on the x-axis. One iteration consists of one invocation of the $n n$ function seen in Figure 20. We consider the cost of fitting $\Theta$ s onto the training and validation sets. The exact formula of the cost function is immaterial to this discussion. It suffices to know that achieving zero cost is not possible, for reasons similar to those that 
explain why our oracle cannot give us perfect predictions. The best possible fit on the validation set would give us a score of approximately 0.23 . The interesting observation that emerges from this figure is the fact that, after a certain number of iterations, we stop seeing any gain of precision on the validation set, while gains are still possible on the training set. Eventually, we observe the cost of the validation set raising slightly. Overfitting is a well-known shortcoming of stochastic optimizations and tends to be mitigated with larger datasets.

\section{CASE STUDY: NON-VOLATILE MEMORY}

Nonvolatile Memory (NVM) is a type of storage memory that preserves data in the absence of power refreshment. Of interest to this article are the variations of NVM that can be used as on-chip memory. The increasing importance of energy in the design of computer architectures has bestowed significant interest onto these kinds of memory technologies, whose examples include Phase-Change RAM (PCRAM), Spin-Transfer Torque RAM (STT-RAM), and Resistive RAM (RRAM). In the absence of memory access operations, the preservation of data does not incur any energy penalty. Nevertheless, said operations, once necessary, tend to be more expensive than what is customarily observed in volatile technologies. Another difference between these two technologies is the fact that in several NVM designs, write operations tend to be more expensive than reads.

Motivated by this last observation, Bouziane et al. (2018) have proposed an NVM optimization based on the elimination of silent stores. This optimization consists of replacing store operations with a sequence of four instructions: (1) a load operation, (2) a comparison, (3) a branch, and (4) the original store itself. Thus, this optimization uses the following code rewriting rule:

$$
* \mathrm{p}=\mathrm{v} \quad \rightarrow \quad \mathrm{u}=* \mathrm{p} ; \mathrm{t}=\mathrm{u}-\mathrm{v} \text {; if }(\mathrm{t}) * \mathrm{p}=\mathrm{v} ; \quad \text { [nvm_opt] }
$$

This rule replaces a store instruction with a test that checks, before updating a memory location, the value already stored there. If the value to be written is already present in memory, then we branch over the store operation. This optimization is beneficial if (1) the execution of the target program presents silent occurrences of store operations and (2) the cost of writing in memory is larger than the cost of the three other instructions necessary to implement the optimization. Bouziane et al. have used a profiler to pinpoint which instructions should be instrumented. Using this approach, they have found benchmarks in the Rodinia collection (Che et al. 2009) for which, under a ratio of $1 / 10$ between read and write operations, the optimization could reduce the cost of memory accesses by up to $42 \%$. In this article, we use our predictor to implement a version of this optimization without profiling support. To this end, we define an optimization strategy as follows:

Definition 5.1 (Optimization Strategy). An optimization strategy is a methodology to rewrite store operations with the [nvm_opt] rule. We consider the following optimization strategies:

- Nothing: the original program, without the application of any rewriting rule

- Everything: the program after every store is rewritten by the [nvm_opt] rule

- Prediction: the program after stores predicted as silent are rewritten by [nvm_opt]

- Optimum: the program in which, given a certain input, every store is instrumented if and only if that instrumentation pays off for that input

We define the cost of memory accesses as a degree of how much they impact the execution of the program. In this article, we adopt a rather abstract notion of cost: it can be a measure of runtime or energy consumption, for instance. If we let $\alpha_{w}$ be the cost of executing one store operation and $\alpha_{r}$ be the cost of executing the three-instruction sequence formed by load, comparison, and branch, then Definition 5.2 gives us the cost of the different optimization strategies. 


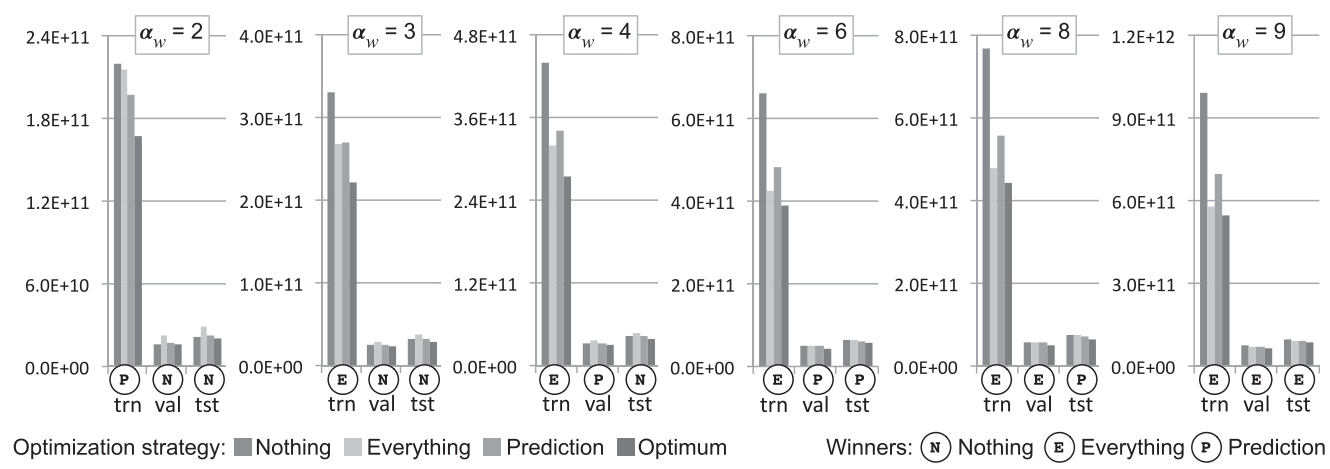

Fig. 23. Comparison between different strategies to implement silent store elimination in nonvolatile memory. The $\mathrm{x}$-axis shows the dataset. The $\mathrm{y}$-axis shows the memory access cost. The predictor used is based on the neural network discussed in Section 4.4.2. The predictor was trained for $P=0.50$. We let $\alpha_{r}=1$ always.

Definition 5.2 (Cost of Memory Accesses). The optimization strategies produce the following cost for one store with ss silent occurrences and $n s$ noisy occurrences ( $s s$ and $n s$ are found via profiling):

- Nothing: $\alpha_{w} \times(s s+n s)$

- Everything: $\alpha_{r} \times(s s+n s)+\alpha_{w} \times n s$

- Prediction: if prediction $=$ silent then $\alpha_{r} \times(s s+n s)+\alpha_{w} \times n s$ else $\alpha_{w} \times(s s+n s)$

- Optimum: let $c_{s}=\alpha_{r} \times(s s+n s)+\alpha_{w} \times n s$ and let $c_{n}=\alpha_{w} \times(s s+n s)$ in $\min \left(c_{s}, c_{n}\right)$

Notice that the so-called optimum strategy cannot be implemented in practice: it involves running the program and then calculating the cost of memory accesses with hindsight. Nevertheless, it gives us a lower bound of what could be expected with very accurate prediction. Figure 23 shows the result of the different optimization strategies applied onto the dataset used in the experiments of Section 4. The dataset is separated into training (27,562 stores), validation, and test (both with 5,906 stores). The figure shows the same experiment, for different values of $\alpha_{w}$, assuming that $\alpha_{r}=1$. We say that an optimization strategy is a winner for a given pair formed by a dataset and some $\alpha_{w}$ if that optimization strategy yields lower memory access cost. The optimum strategy is not considered in this definition; otherwise, it would be always the winner. Winners vary according to the ratio $\alpha_{w} / \alpha_{r}$ and according to the test set. In Figure 23, every strategy won at least once for each dataset. The only exception is Nothing, which never won for the training set.

The Prediction strategy tends to be the best choice for ratios of $\alpha_{w} / \alpha_{r}$ between 4 and 8 in Figure 23. However, the space for gains is small. As already extensively discussed, most of the stores are noisy; hence, we are left with about $20 \%$ of instructions silent more than $50 \%$ of the time. The neural network can correctly pinpoint approximately two-thirds of these stores, which we rewrite using [nvm_opt]. This optimization pays off when the cost of the rewriting sequence is less than eight times the cost of the memory update. If this ratio is higher, then the false negatives that we miss (stores that were marked as noisy but end up being silent) start increasing too much the cost of memory accesses. If we set our predictor to $P=0.50$, e.g., "find which stores are silent more than $50 \%$ of the time," then, at a ratio $\alpha_{w} / \alpha_{r}=2$, prediction yields an overall access cost that is $9 \%$ lower than the Everything strategy for the training set. At $\alpha_{w} / \alpha_{r}=3$, they produce approximately equal results. Finally, at $\alpha_{w} / \alpha_{r}=4$, Everything is $5 \%$ better.

We emphasize that results vary per benchmark, and it is not difficult to find cases in which Prediction is consistently better than the other optimization strategies for very large ratios $\alpha_{w} / \alpha_{r}$. Figure 24 supports this statement: it shows how the different optimization strategies behave on 


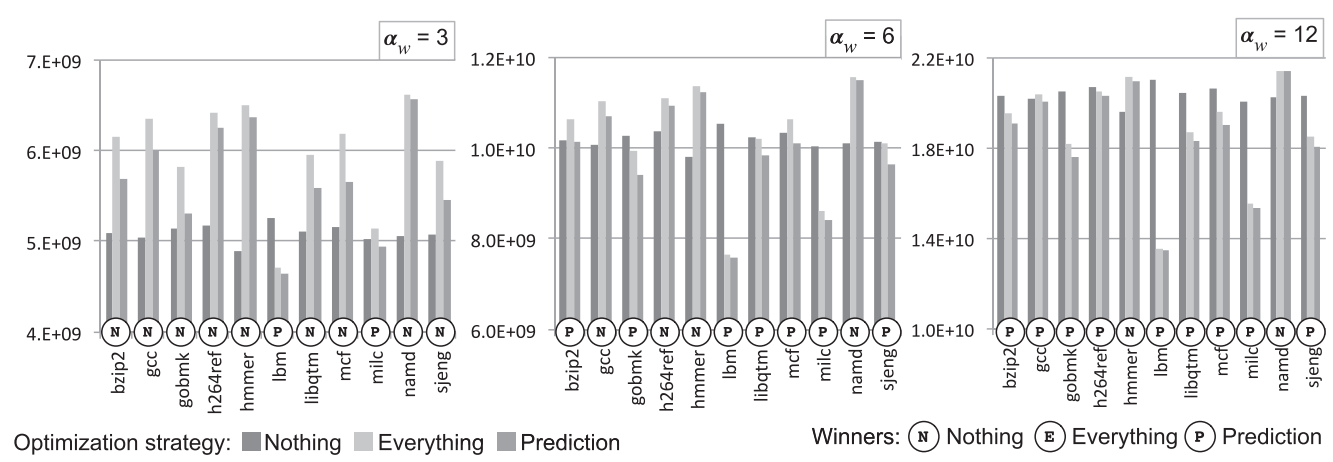

Fig. 24. Different strategies to implement silent store elimination in nonvolatile memory on SPEC CPU2006 CINT. The $x$-axis shows benchmarks. The $y$-axis shows memory access cost. The predictor is the neural network with $P=0.50$. Benchmarks gcc, gobmk, and namd were not used in the training set.

the integer programs in SPEC CPU2006. We have eliminated perlbench from this figure, because it has a very small number of dynamic store instances compared to the other benchmarks. ${ }^{3}$ At $\alpha_{w} / \alpha_{r}=12$, Prediction beats Nothing in every benchmark, except hmmer and namd. At $\alpha_{w} / \alpha_{r}=$ 15 , a result not shown in the figure, but Prediction is the winner for all of them, including those two. For the programs in Figure 24, the Everything strategy will start to become profitable only for very high-and unrealistic-values of $\alpha_{w} / \alpha_{r}$. For instance, for SPEC's gcc, this ratio is $\alpha_{w} / \alpha_{r} \approx 165,000$.

\section{RELATED WORK}

Silent stores are a well-known phenomenon in the field of computer architecture, coming to widespread attention in the early 2000s due to the work of Kevin Lepak and his collaborators (Bell et al. 2000; Lepak and Lipasti 2000a, 2000b, 2002). Much of this attention was inspired by even earlier results, due to Lipasti et al., showing that values tend to have strong locality and can be predicted with relatively high accuracy in hardware (Lipasti and Shen 1996; Lipasti et al. 1996). These preliminary forays in the study of value recurrence, which include the notion of silent store, were very influential, having motivated a long string of research efforts, among which this article is included. Nevertheless, to the best of our knowledge, there has not been any attempt to predict silent stores statically-the goal of this article. Instead, researchers use profiling, be it implemented in software (Khan et al. 2017; Wen et al. 2017, 2018) or in hardware, like in the early days of research in this field (Butts and Sohi 2002; Lepak and Lipasti 2000a, 2000b).

Previous Work on the Characterization of Silent Stores. The work that is the closest to ours is the study carried out by Bell et al. (2000). Bell et al. have analyzed the behavior of store instructions in 16 large benchmarks available in SPEC CPU95. Some of the facts observed in that work have been observed in our experiments; however, there are also conclusions that are different. Notably, in their study, Bell et al. have found a higher proportion of silent stores. Out of the 16 benchmarks considered, only three presented less than $19 \%$ of silent writes, whereas this is the average of silentness among our benchmarks. We speculate that one of the reasons behind such difference is the fact that Bell et al. have analyzed not only visible but also invisible instructions (as defined in Section 2.3), whereas we only analyze instructions available in the source code. For the sake of comparison, we discuss some of the results published by Bell et al.:

\footnotetext{
${ }^{3}$ Perlbench presents $91,439,220$ dynamic store instances. The integer benchmark in SPEC CPU2006 with the second lowest number of dynamic stores is Ibm, with 1,754,320,000 instances: almost 20 times more.
} 
- "Integer and floating point zero occurs very often [among dynamic instances of silent stores]." Our findings corroborate this conclusion. Figure 7 shows that the presence of zero in the list of dependences of a store, or as the last value to be stored, increases by a wide margin the chance of silentness. Furthermore, Figure 3 indicates that Ozr is one of the features with the highest probability of leading to silentness.

- "In all of the integer benchmarks, less than 25\% of all static silent stores contribute over $90 \%$ of the total silent stores dynamically executed." We have also observed this irregularity. We believe that this distribution is a natural consequence of the well-known Pareto principle that governs the execution of programs: most of their execution is due to a few instructions.

- "In general, stores that are not executed many times have a decreased probability of being silent." We failed to observe this result. As evidence to the contrary, the curves in Figures 11 and 12 tend to be very similar, but the latter only includes stores with at least 50 dynamic instances. Furthermore, the nesting depth of loops, alone, does not seem to cause a noticeable difference in the chance of silentness, as features S10, S11, S12, and S13 in Figure 3 imply.

Nevertheless, in spite of similarities, our work is very different than that of Bell et al. They analyze the dynamic behavior of store operations but do not attempt to predict it. We focus on the static characteristics of instructions that might cause them to be silent. Our goal is to predict the likelihood that a store operation is silent. In the effort to achieve this goal, we provide insights about the nature of silent stores. Some of these insights were present in Bell's work; others were not.

On the Application of Silent Stores. Section 5 presents an application of our predictor; however, silent store optimization is not a focus of this article. Nevertheless, much work has been done along this direction. Different research groups have designed new hardware that capitalizes on the silent store phenomenon with great profit. On-the-flight detection of silent stores enables the construction of faster hardware pipelines (Lepak and Lipasti 2000a; Subramaniam and Loh 2006), as it shortens the waiting time due to data dependencies. Dusser et al. (2009) have shown how to boost the performance of cache systems by exploring silentness. Rajwar and Goodman (2001) rely on the existence of silent stores to explore speculative parallelization more extensively. This previous body of literature proposed the detection of silent stores at the hardware level. Recently, profilingbased detection of silent stores has been demonstrated to be effective and useful. Profiling supports the discovery of performance bugs in software (Wen et al. 2017, 2018) and the elimination of false dependencies that hinder parallelism (Chabbi et al. 2018) or the style of memory optimization that we have described in Section 5 (Bouziane et al. 2018). We believe that static prediction complements such previous work, easing the burden on dynamic detectors and enhancing the ability of profilers to provide meaningful reports to users.

\section{CONCLUSION AND FUTURE WORK}

This article has presented a study on the influence of syntactic program features on the occurrence of silent stores. From this study, we have derived predictors that determine store instructions that tend to be silent during the execution of programs. In the effort to build such predictors and understand the behavior of store operations, we have collected a large volume of data, which has given us the opportunity to reach interesting conclusions, some of which we list below:

- Syntax that might cause the deposit of the value zero or a Boolean value in memory is the most consistent source of silentness. In the opposite direction, increments and nonzero constants tend to lead to noisy stores. Data in Figure 3 substantiate this claim. 
- The probability that a store is silent or noisy follows a U-shaped distribution, as Figure 13 shows. In other words, silent stores are very silent, and noisy stores are very noisy. A consequence of this observation is that if a store is observed to be silent or noisy, then this behavior is likely to repeat if the same instruction is executed.

- The distribution of silent stores among benchmarks seems to follow a normal distribution, as Figure 10 indicates. This observation can be used to detect anomalies, such as performance bugs. For instance, a program can be considered suspicious if it contains a number of silent stores above a threshold $P$ falling outside two or three standard deviations of $P$ 's distribution.

- Our choice of features leads to the construction of static predictors whose precision is significantly (in a statistical sense) superior to trivial prediction strategies. Figure 16 provides visual evidence of this claim.

Much can be done out of the insights presented in this work. Our data (available at http:// www.lirmm.fr/continuum-project/pages/s3a.html) can be used in the construction of tools able to detect performance bugs or carry out silent store optimizations. Our methodology can be further expanded via the inclusion of different program features or more benchmarks. In particular, we have restricted our attention to benchmarks written in C. It might be an interesting project to analyze the occurrence of silent stores in benchmarks written in other programming languages.

\section{ACKNOWLEDGMENTS}

We thank the referees of ACM TACO for many insightful comments and suggestions that greatly contributed to improve this work. We also thank Breno Campos and Marcos Siraichi for reading an early draft of this article.

\section{REFERENCES}

Rakesh Agrawal and Ramakrishnan Srikant. 1994. Fast algorithms for mining association rules in large databases. In VLDB. Morgan Kaufmann Publishers, San Francisco, 487-499.

Thomas Ball and James R. Larus. 1993. Branch prediction for free. In PLDI. ACM, New York, 300-313.

Gordon B. Bell, Kevin M. Lepak, and Mikko H. Lipasti. 2000. Characterization of silent stores. In PACT. IEEE, 133.

Rabab Bouziane, Erven Rohou, and Abdoulaye Gamatié. 2018. Compile-time silent-store elimination for energy efficiency: An analytic evaluation for non-volatile cache memory. In RAPIDO. HiPEAC, Manchester, 1-8.

Jeffrey Adam Butts and Guri Sohi. 2002. Dynamic dead-instruction detection and elimination. In ASPLOS. ACM, New York, 199-210.

Augustin Louis Cauchy. 1847. Méthode Générale pour la résolution des systèmes d'Équations simultanées. Comptes Rendus Hebd. Séances Acad. Sci. 25, 10 (1847), 536-538.

Milind Chabbi, Shasha Wen, and Xu Liu. 2018. Featherlight on-the-fly false-sharing detection. In PPoPP. ACM, New York, 152-167.

Shuai Che, Michael Boyer, Jiayuan Meng, David Tarjan, Jeremy W. Sheaffer, Sang-Ha Lee, and Kevin Skadron. 2009. Rodinia: A benchmark suite for heterogeneous computing. In IISWC. IEEE, 44-54.

David R. Cox. 1958. The regression analysis of binary sequences (with discussion). J. Roy. Stat. Soc. B 20 (1958), 215-242.

Ron Cytron, Jeanne Ferrante, Barry K. Rosen, Mark N. Wegman, and F. Kenneth Zadeck. 1991. Efficiently computing static single assignment form and the control dependence graph. ACM Trans. Program. Lang. Syst. 13, 4 (1991), 451-490.

Arthur P. Dempster. 1967. Upper and lower probabilities induced by a multivalued mapping. Ann. Math. Stat. 38, 2 (1967), 325-339.

Julien Dusser, Thomas Piquet, and André Seznec. 2009. Zero-content augmented caches. In ICS. ACM, New York, 46-55.

Geoffrey W. Hill. 1970. Algorithm 395: Student's T-distribution. Commun. ACM 13, 10 (1970), 617-619.

Samira Khan, Chris Wilkerson, Zhe Wang, Alaa R. Alameldeen, Donghyuk Lee, and Onur Mutlu. 2017. Detecting and mitigating data-dependent DRAM failures by exploiting current memory content. In MICRO. ACM, New York, 27-40.

Chris Lattner and Vikram Adve. 2004. LLVM: A compilation framework for lifelong program analysis \& transformation. In CGO. IEEE, 75-85.

Guilherme V. Leobas, Breno C. F. Guimarães, and Fernando M. Q. Pereira. 2018. More than meets the eye: Invisible instructions. In SBLP. ACM, New York, 27-34. 
Kevin M. Lepak and Mikko H. Lipasti. 2000a. On the value locality of store instructions. In ISCA. ACM, New York, $182-191$. Kevin M. Lepak and Mikko H. Lipasti. 2000b. Silent stores for free. In MICRO. ACM, New York, 22-31.

Kevin M. Lepak and Mikko H. Lipasti. 2002. Temporally silent stores. In ASPLOS. ACM, New York, 30-41.

Mikko H. Lipasti and John Paul Shen. 1996. Exceeding the dataflow limit via value prediction. In MICRO. IEEE, 226-237.

Mikko H. Lipasti, Christopher B. Wilkerson, and John Paul Shen. 1996. Value locality and load value prediction. In ASPLOS. ACM, New York, 138-147.

Chi-Keung Luk, Robert Cohn, Robert Muth, Harish Patil, Artur Klauser, Geoff Lowney, Steven Wallace, Vijay Janapa Reddi, and Kim Hazelwood. 2005. Pin: Building customized program analysis tools with dynamic instrumentation. In PLDI. ACM, New York, 190-200.

Sparsh Mittal and Jeffrey S. Vetter. 2016. A survey of software techniques for using non-volatile memories for storage and main memory systems. Trans. Parallel Distrib. Syst. 27, 5 (2016), 1537-1550.

Fernando Magno Quintao Pereira, Raphael Ernani Rodrigues, and Victor Hugo Sperle Campos. 2013. A fast and lowoverhead technique to secure programs against integer overflows. In CGO. IEEE, 1-11.

Ravi Rajwar and James R. Goodman. 2001. Speculative lock elision: Enabling highly concurrent multithreaded execution. In MICRO. IEEE, 294-305.

Glenn Shafer. 1976. A Mathematical Theory of Evidence. Princeton University Press, Princeton, NJ.

Sam S. Stone, Kevin M. Woley, and Matthew I. Frank. 2005. Address-indexed memory disambiguation and store-to-load forwarding. In MICRO. IEEE, 171-182.

Samantika Subramaniam and Gabriel H. Loh. 2006. Fire-and-forget: Load/store scheduling with no store queue at all. In MICRO. IEEE, 273-284.

Shasha Wen, Milind Chabbi, and Xu Liu. 2017. REDSPY: Exploring value locality in software. In ASPLOS. ACM, New York, $47-61$.

Shasha Wen, Xu Liu, John Byrne, and Milind Chabbi. 2018. Watching for software inefficiencies with witch. In ASPLOS. ACM, New York, 332-347.

Youfeng Wu and James R. Larus. 1994. Static branch frequency and program profile analysis. In MICRO. ACM, New York, 1-11.

Ping Zhou, Bo Zhao, Jun Yang, and Youtao Zhang. 2009. Energy reduction for STT-RAM using early write termination. In ICCAD. ACM, New York, 264-268.

Received May 2018; revised August 2018; accepted September 2018 